\title{
On the Performance of CUSUM control charts for monitoring the Coefficient of Variation with Measurement Errors
}

\author{
Kim Phuc Tran • Huu Du Nguyen • Phuong
}

Hanh Tran • Cédric Heuchenne

Received: date / Accepted: date

\begin{abstract}
In this paper, we investigate the effect of the measurement error on the performance of cumulative sum (CUSUM) control charts monitoring the coefficient of variation. The measurement errors are supposed to follow a linear covariate error model. The obtained results show that the precision error ratio and the accuracy error have negative impact on the chart performance. Moreover, in order to overcome the difficulty in predetermining a specific value for the process shift size, we suggest to optimize parameters of the charts according to the random shift size in a given interval. The robustness of the proposed method is studied. An example is given to illustrate the use of the CUSUM charts on a real quality control problem from sintering process.
\end{abstract}

Keywords SPC; Measurement Error; CUSUM Control charts; Coefficient of Variation; Markov Chain

K. P. Tran

Ecole Nationale Supérieure des Arts et Industries Textiles, GEMTEX Laboratory, 59056 Roubaix, France

E-mail: kim-phuc.tran@ensait.fr

H. D. Nguyen

Division of Artificial Intelligence, Dong A University, Danang, Vietnam

E-mail: dunh@donga.edu.vn

P.H. Tran

HEC Liège, Management School of the University of Liège, Liège, Belgium

E-mail: tran@student.uliege.be

C. Heuchenne

HEC Liège, Management School of the University of Liège, Liège, Belgium

E-mail: C.Heuchenne@ulg.ac.be 
Acronyms

$\begin{array}{ll}\text { ARL } & \text { Average Run Length } \\ \text { CUSUM } & \text { Cumulative Sum } \\ \text { CV } & \text { Coefficient of Variation } \\ \text { EARL } & \text { Expected Average Run Length } \\ \text { EWMA } & \text { Exponentially Weighted Moving Average } \\ \text { SPC } & \text { Statistical Process Control }\end{array}$

\section{Introduction}

Control chart is a very efficient tool in statistical process control (SPC) to detect timely deviations in a production process that may lead to defective items. A signal from the control chart indicates the appearance of assignable causes that need to be fixed to ensure the process stability. Thence, it allows to eliminate waste and reduces production costs.

In general, a control chart contains a central line and the control limits. The process is monitored by sampling, calculating the quality of interest, and plotting this value on the chart. If the sample point is within the control limit, the process is said to be in-control. Otherwise, it is said to be out-of-control and the assignable causes should be considered and removed. During the last decade, a large number of new advanced control charts has been introduced and they have been widely applied in many fields of industrial manufacturing.

The coefficient of variation $(\mathrm{CV})$ is an important quality characteristic that has several applications in applied statistics and SPC. It is defined as a ratio of the standard deviation $\sigma$ to the mean $\mu$ of a probability distribution. In many processes, the mean and the variance of the quantity of interest do not need to be constant or to be independent from others. In fact, the variance of this quantity could be a function of its mean while the mean itself varies from time to time. Therefore, the CV should be a characteristic of interest: no matter how the mean or the variance of the quantity is, the process is still considered as in-control as long as its CV remains stable. Examples of using CV charts can be seen in various fields such as materials engineering and manufacturing, textile industry, and chemical and biological quality control, see Castagliola et al. ${ }^{1}$.

In the SPC literature, the problem of monitoring the CV was initiated by Kang et al. ${ }^{2}$ with a Shewhart control chart. Then, it was developed with the exponentially weighted moving average (EWMA) control chart (Castagliola et al. ${ }^{1}$ ), the synthetic control chart (Calzada and Scariano ${ }^{3}$ ), the Run Rules control chart (Castagliola et al. ${ }^{4}$ ), the variable sampling interval control chart (Castalgiola et al. ${ }^{5}$ ), the variable sample size control chart (Amdouni et al. ${ }^{6}$; Castagliola et al. ${ }^{7}$ ), and the cumulative sum (CUSUM) control chart (Tran and $\operatorname{Tran}^{8}$ ). Other control charts monitoring the $\mathrm{CV}$ can be seen in Jian et al. ${ }^{9}$ and Abbasi and Adegoke ${ }^{10}$. 
An important problem shoud be considered in designing a control chart is the existence of the measurement error. In fact, many control charts are desinged under the assumption that there is no measurement error. This assumption, however, may not be true in practice. Ignoring the presence of the measurement error may lead to the misunderstanding about the statistical properties of the desinged control charts. Because of this fact, the effect of the measurement error has been studied by a number of authors, for example Linna and Woodall ${ }^{11}$; Maravelakis ${ }^{12}$; Hu et al. ${ }^{13}$; Noorossana and Zerehsaz ${ }^{14}$, and Tran et al. ${ }^{15}$. In the recent control charts monitoring the CV, this problem has been also studied. The first study was perhaps conducted by Yeong et al. ${ }^{16}$ with a Shewhart chart to monitor the CV in the presence of the measurement error using a linear covariate error model. In this study, the author used a tight assumption that the relation between the in-control CV and the out-ofcontrol CV is independent from the measurment error. This assumption was then eliminated in the design of other CV control charts considering the measurement error, see, for example Tran et al. ${ }^{17}$, Tran et al. ${ }^{18}$, Nguyen et al. ${ }^{19}$, and Tran and Heuchenne ${ }^{20}$.

It is desirable in practice to design a control chart with high efficiency in detecting the process shift. Among several control charts monitoring the CV, the CUSUM control chart brings a better statistical performance compared to the others (Tran and $\operatorname{Tran}^{8}$ ). Therefore, the purpose of this study is to investigate the effect of the measurement error on the CUSUM control chart monitoring the $\mathrm{CV}$ using the linear covariate error model as suggested by Linna and Woodall ${ }^{11}$. According to the discussion in Nguyen et al. ${ }^{19}$, we are going to monitor the CV squared with two one-sided CUSUM charts, denoted by CUSUM- $\gamma^{2}$, instead of monitoring directly the CV. In addition, in order to overcome the difficulty of predetermining a specific shift size, we suggest to design the CUSUM chart with parameters optimized based on a possible interval of the process shift. In particular, in this study we want to investigate

- the impact of the measurement error on the performance of the CUSUM- $\gamma^{2}$ control charts,

- the performance of the CUSUM- $\gamma^{2}$ control charts with parameters optimized based on the random shift size in a given interval.

The rest of the paper is organized as follows: in Section 2, we introduce briefly the linear covariate error model for the $\mathrm{CV}$ as well as the distribution of the CV squared in the presence of the measurement error. The implementation of the two one-sided CUSUM- $\gamma^{2}$ control charts in the presence of the measurement error is presented in Section 3. Section 4 is devoted to analyzing the effect of measurement errors on the charts performance. Section 5 provides an illustrative example of the use of the proposed charts. Some suggestions and remarks are given to conclude in Section 6. 


\section{Linear covariate error model for the coefficient of variation}

In this section, we present briefly the linear covariate error model for the $\mathrm{CV}$ as suggested in Linna and Woodall ${ }^{11}$. Suppose that a set of $n$ samples, $\left\{X_{i, 1}, X_{i, 2}, \ldots, X_{i, n}\right\}$, is taken to monitor the quality of interst $X$ in which the index $i$ stands for the consecutive times of measuring, $i=1,2, \ldots$. Moreover, it is assumed that $X_{i, j}$ are independent identically distributed (i.i.d) from a normal distribution, $X_{i, j} \sim N\left(\mu_{0}+a \sigma_{0},\left(b \sigma_{0}\right)^{2}\right)$. The parameters $a$ and $b$ represent the mean shift and the standard deviation shift of the process. If $a \neq 0$ or $b \neq 1$, the process has been shifted; on the contrary, the process is in-control.

In practice, because of the measurement error the true values $\left\{X_{i, 1}, X_{i, 2}, \ldots\right.$, $\left.X_{i, n}\right\}$ are not observable. Instead, we can only observe $\left\{X_{i, j, 1}^{*}, X_{i, j, 2}^{*}, \ldots, X_{i, j, m}^{*}\right\}$, $m \geqslant 1$, where $X_{i, j, k}^{*}$ is the $\mathrm{k}^{\text {th }}$ measurement of the item $j$ at the $i$ sampling and the symbol "**" is to imply the actually observed values. Linna and Woodall ${ }^{11}$ proposed to use the following linearly covariate error model:

$$
X_{i, j, k}^{*}=A+B X_{i, j}+\varepsilon_{i, j, k},
$$

where the constants $A$ and $B$ are well-known estimated from phase I data, $\varepsilon_{i, j, k}$ is a normal $\left(0, \sigma_{M}\right)$ random error representing the measurement inaccuracy, which is independent of $X_{i, j}$.

Let $\bar{X}_{i, j}^{*}$ be the mean of $m$ observed quantities of the same item $j$ at the $\mathrm{i}^{\text {th }}$ sampling, then the distribution of $\bar{X}_{i, j}^{*}$ can be obtained as

$$
\bar{X}_{i, j}^{*} \sim N\left(\mu^{*}, \sigma^{* 2}\right)=N\left(A+B\left(\mu_{0}+a \sigma_{0}\right), B^{2} b^{2} \sigma_{0}^{2}+\frac{\sigma_{M}^{2}}{m}\right) .
$$

Let us denote $\eta=\frac{\sigma_{M}}{\sigma_{0}}$ (the precision error ratio), $\theta=\frac{A}{\mu_{0}}$ (the accuracy error), and $\gamma_{0}=\frac{\sigma_{0}}{\mu_{0}}$ (the in-control CV value). The CV of $\bar{X}_{i, j}^{*}$ is therefore

$$
\gamma^{*}=\frac{\sqrt{B^{2} b^{2} \sigma_{0}^{2}+\frac{\sigma_{M}^{2}}{m}}}{A+B\left(\mu_{0}+a \sigma_{0}\right)}=\frac{\sqrt{B^{2} b^{2}+\frac{\eta^{2}}{m}}}{\theta+B\left(1+a \gamma_{0}\right)} \times \gamma_{0},
$$

Let $\tau$ denote the shift size, i.e. $\gamma_{1}=\tau \gamma_{0}$ where $\tau_{1}$ is the out-of-control value of the CV. It was shown in Nguyen et al. ${ }^{19}$ that

$$
\gamma_{1}^{*}=\frac{\sqrt{B^{2} b^{2}+\frac{\eta^{2}}{m}}}{\theta+\frac{B b}{\tau}} \times \gamma_{0}
$$

The sample $\mathrm{CV} \hat{\gamma}_{i}^{*}$ in the presence of the measurement error is defined as

$$
\hat{\gamma}_{i}^{*}=\frac{S_{i}^{*}}{\overline{\bar{X}}_{i}^{*}},
$$

in which $\bar{X}_{i}^{*}$ and $S_{i}^{*}$ are the sample mean and the sample standard deviation of $\bar{X}_{1, j}^{*}, \ldots, \bar{X}_{n, j}^{*}$. 
The distribution of the sample CV of normal variables has been studied by many authors, for example Iglewicz and Myers ${ }^{25}$; Reh and Scheffler ${ }^{26}$; Van$\mathrm{gel}^{27}$. In this paper, we adopt an approximation for the cumulative distribution function (c.d.f.) of the CV squared suggested by Castagliola et al. ${ }^{1}$ as

$$
F_{\hat{\gamma}^{* 2}}\left(x \mid n, \gamma^{*}\right)=1-F_{F}\left(\frac{n}{x} \mid 1, n-1, \frac{n}{\gamma^{* 2}}\right)
$$

where $F_{F}($.$) is the c.d.f. of the noncentral F$ distribution and the parameter $\gamma^{*}$ is computed from (4).

\section{Implementation of the CUSUM- $\gamma^{2}$ control charts with measurement errors}

Denote $\mu_{0}\left(\hat{\gamma}^{* 2}\right)$ and $\sigma_{0}\left(\hat{\gamma}^{* 2}\right)$ the mean and the standard deviation of the sample $\gamma^{* 2}$ when the process is in-control. There is no closed form for these quantities from the literature. We then apply an accurate approximations provided by Breunig $^{21}$ as:

$\mu_{0}\left(\hat{\gamma}^{* 2}\right)=\gamma_{0}^{* 2}\left(1-\frac{3 \gamma_{0}^{* 2}}{n}\right)$,
$\sigma_{0}\left(\hat{\gamma}^{* 2}\right)=\sqrt{\gamma_{0}^{* 4}\left(\frac{2}{n-1}+\gamma_{0}^{* 2}\left(\frac{4}{n}+\frac{20}{n(n-1)}+\frac{75 \gamma_{0}^{* 2}}{n^{2}}\right)\right)-\left(\mu_{0}\left(\hat{\gamma}^{* 2}\right)-\gamma_{0}^{* 2}\right)^{2}}$,

where $\gamma_{0}^{*}$ is defined in (3) with $a=0$ and $b=1$.

Similar to Tran and $\operatorname{Tran}^{8}$, the two separated one-sided CUSUM control charts for monitoring the CV squared are explored in this paper. More detail of the reason are discussed in Castagliola et al. ${ }^{1}$. In particular, the proposed one-sided CUSUM-CV charts in the presence of measurement errors are as follows:

- an upward CUSUM chart (denoted by "upward CUSUM- $\gamma^{2}$ ") corresponding to an upper control limit $H^{+}=h^{+} \mu_{0}\left(\hat{\gamma}^{* 2}\right)$ to detect the increase in the $\mathrm{CV}$,

$$
C_{i}^{+}=\max \left\{0, C_{i-1}^{+}+\left(\hat{\gamma}_{i}^{* 2}-\mu_{0}\left(\hat{\gamma}^{* 2}\right)-K^{+}\right\},\right.
$$

where $K^{+}=k^{+} \sigma_{0}\left(\hat{\gamma}^{* 2}\right)$ and $C_{0}^{+}=0$,

- a downward CUSUM chart (denoted as "downward CUSUM- $\gamma^{2}$ ") corresponding to the lower control limit $H^{-}=h^{-} \mu_{0}\left(\hat{\gamma}^{* 2}\right)$ to detect the decrease in the $\mathrm{CV}$,

$$
C_{i}^{-}=\max \left\{0, C_{i-1}^{-}-\left(\hat{\gamma}_{i}^{* 2}-\mu_{0}\left(\hat{\gamma}^{* 2}\right)-K^{-}\right\},\right.
$$

where $K^{-}=k^{-} \sigma_{0}\left(\hat{\gamma}^{* 2}\right)$ and $C_{0}^{-}=0$. 


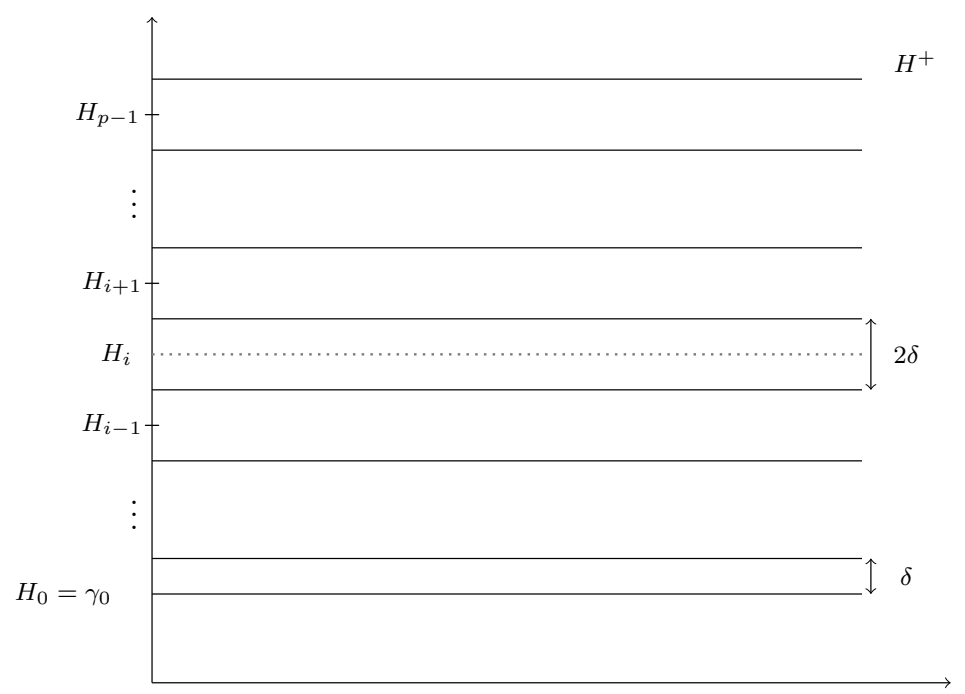

Fig. 1: Control limit interval of upward chart devided into $p-1$ subintervals of width $2 \delta$ and first interval of with $\delta$.

The parameters $\left(k^{+}, h^{+}\right)$and $\left(k^{-}, h^{-}\right)$are called the control coefficients of the upward and the downward CUSUM- $\gamma^{* 2}$ control chart, respectively. The control charts are designed by finding these coefficients.

In order to evaluate the performance of the proposed chart, we use the average run length $(A R L)$, which is the average number of samples before the first out-of-control point is plotted in the control chart, see Tran et al. ${ }^{22}$ for more details. A general approach to calculate the $A R L$ is to use the Markov chain method suggested by Brook and Evans ${ }^{23}$. The method is described in the sequel.

Firstly, the limit interval of upward (downward) chart is divided into $p$ sub-intervals in which the first sub-interval is $\delta=\frac{H^{+}}{2 p-1}\left(\delta=\frac{H^{-}}{2 p-1}\right)$ in width and the others are $2 \delta$ in width. Figure 1 demonstrates this subdivision for the upward chart. In this figure, each sub-interval $\left(H_{j}-\delta, H_{j}+\delta\right]$ represents a transient state of the Markov chain, where $H_{j}$ is the midpoint of the subinterval $j, j=0, . ., p-1$; the states 0 to $p-1$ are in-control states while the state $N$ is out-of-control. When $C_{i}^{+}$(or $\left.C_{i}^{-}\right) \in\left(H_{j}-\delta, H_{j}+\delta\right]$, the Markov chain is in the transient sate $j$ for sample $i$; if not, the chain reaches an absorbing state. $p$ is choosen sufficiently large so that $H_{j}$ can be considered as an approximately representative value of the state $j$ ( $p$ is set to be 200 in this paper). In this subdivision, the zero state has half size of the others, leading to better Markov chain approximation with the same width of sub-intervals as in Castagliola et al. ${ }^{1}$. 
The transition probability matrix $\mathbf{P}$ of the discrete-time Markov chain is

$$
\mathbf{P}=\left(\begin{array}{cc}
\mathbf{Q} & \mathbf{r} \\
\mathbf{0}^{T} & 1
\end{array}\right)=\left(\begin{array}{ccccc}
Q_{0,0} & Q_{0,1} & \ldots & Q_{0, p-1} & r_{0} \\
Q_{1,0} & Q_{1,1} & \cdots & Q_{1, p-1} & r_{1} \\
\vdots & \vdots & & & \vdots \\
Q_{p-1,0} & Q_{p-1,1} & \ldots & Q_{p-1, p-1} & r_{p-1} \\
0 & 0 & \ldots & 0 & 1
\end{array}\right)
$$

where $\mathbf{Q}$ is the $(p, p)$ matrix of transient probabilities, $\mathbf{0}=(0,0, \ldots, 0)^{T}$ and $\mathbf{r}$ is $p$-vector statisfying $\mathbf{r}=(1-\mathbf{Q} \mathbf{1})$ (i.e., row probabilities must sum to 1 ) with $\mathbf{1}=(1,1, \ldots, 1)^{T}$.

Then, the elements $Q_{i, j}$ of the matrix $\mathbf{Q}$ is calculated by the following formulas:

- for the upward chart,

$$
\begin{aligned}
Q_{i, 0}= & F_{\hat{\gamma}^{* 2}}\left(\mu_{0}\left(\hat{\gamma}^{* 2}\right)-H_{i}+K^{+}+\delta \mid n, \gamma_{1}^{*}\right) \text { and } \\
Q_{i, j}= & F_{\hat{\gamma}^{* 2}}\left(\mu_{0}\left(\hat{\gamma}^{* 2}\right)+H_{j}-H_{i}+\delta+K^{+} \mid n, \gamma_{1}^{*}\right) \\
& -F_{\hat{\gamma}^{* 2}}\left(\mu_{0}\left(\hat{\gamma}^{* 2}\right)+H_{j}-H_{i}-\delta+K^{+} \mid n, \gamma_{1}^{*}\right) ;
\end{aligned}
$$

- for the downward chart,

$$
\begin{aligned}
Q_{i, 0}= & 1-F_{\hat{\gamma}^{* 2}}\left(\mu_{0}\left(\hat{\gamma}^{* 2}\right)+H_{i}-K^{-}-\delta \mid n, \gamma_{1}^{*}\right) \text { and } \\
Q_{i, j}= & F_{\hat{\gamma}^{* 2}}\left(\mu_{0}\left(\hat{\gamma}^{* 2}\right)+H_{j}-H_{i}+\delta+K^{+} \mid n, \gamma_{1}^{*}\right) \\
& -F_{\hat{\gamma}^{* 2}}\left(\mu_{0}\left(\hat{\gamma}^{* 2}\right)+H_{j}-H_{i}-\delta+K^{+} \mid n, \gamma_{1}^{*}\right),
\end{aligned}
$$

where $F_{\hat{\gamma}^{* 2}}($.$) is the c.d.f. of \hat{\gamma}^{* 2}$ in (6).

Let $\mathbf{q}$ be the $(p-1,1)$ vector of initial probabilities associated with the $p$ transient states, i.e., $\mathbf{q}=\left(q_{0}, q_{1}, \ldots, q_{p-1}\right)^{T}$. The zero-state $A R L$ of performance is corresponding to the "restart state" of initial state, namely $\mathbf{q}=$ $(1,0, \ldots, 0)$.

The $A R L$ value of the CUSUM- $\gamma^{2}$ control chart is then calculated by

$$
A R L=\mathbf{q}^{T}(\mathbf{I}-\mathbf{Q})^{-1} \mathbf{1}
$$

The design of the CUSUM- $\gamma^{2}$ control charts now is to find the chart coefficients $\left(k^{+}, h^{+}\right)$or $\left(k^{-}, h^{-}\right)$such that they optimize the chart's performance. In general, these values are found to satisfy two constraints: (1) the in-control value $A R L_{0}$ is equal to a predetermined desirable value and (2) the out-ofcontrol value $A R L_{1}$ is minimized.

It should be considered that this procedure is based on the $A R L$, which is a function of the shift size $\tau$. Thus, to design the CUSUM- $\gamma^{2}$ control chart, the quality practitioners need to predict a possibly specific value for $\tau$ and then calculate the corresponding optimal values $\left(k^{*+}, h^{*+}\right)$ or $\left(k^{*-}, h^{*-}\right)$ that satisfy two constraints mentioned above. However, it is not possible in practice to give an exact prediction for the shift size because (1) without related 


\begin{tabular}{c|c|cccccc}
\hline$\tau_{1}$ & $\tau_{2}$ & $\eta=0$ & $\eta=0.1$ & $\eta=0.2$ & $\eta=0.3$ & $\eta=0.5$ & $\eta=1$ \\
\hline \multirow{3}{*}{0.95} & 0.5 & 179.09 & 178.88 & 178.88 & 179.13 & 179.39 & 179.90 \\
& 0.7 & 93.41 & 93.31 & 93.27 & 93.44 & 93.51 & 93.68 \\
& 0.8 & 32.62 & 32.57 & 32.54 & 32.63 & 32.65 & 32.68 \\
\hline \multirow{3}{*}{1.05} & 1.3 & 25.08 & 25.07 & 25.08 & 25.09 & 25.06 & 25.00 \\
& 1.5 & 52.87 & 52.86 & 52.86 & 52.87 & 52.80 & 52.59 \\
& 2.0 & 72.59 & 72.58 & 72.57 & 72.57 & 72.44 & 72.01 \\
\hline
\end{tabular}

Table 1: Relative errors $\Delta_{1}$ betwwen the $A R L$ corresponding to the parameters optimized from the predicted shift size $\left(\tau_{1}\right)$ and the actual shift size $\left(\tau_{2}\right)$ under the presence of the measurement error with $B=1, m=1, \theta=0.05$ and $n=5$.

historical data they have no information about the entity of next shift size, and (2) the shift size is usually unstable: it varies from time to time and from process to process. If the predetermined value of $\tau$, say $\tau_{1}$, is different from the true shift, say $\tau_{2}$, the run-length properties of the designed control chart will be seriously affected. Table 1 provides an illustrative example for this problem where the value

$$
\Delta_{1}=\frac{A R L_{\tau_{1}}-A R L_{\tau_{2}}}{A R L_{\tau_{2}}}
$$

is presented. By its definition, $\Delta_{1}$ represents the relative error between the $A R L$ corresponding to the control coefficients optimized from a predicted shift $\tau_{1}$, denoted by $A R L_{\tau_{1}}$, and the $A R L s$ corresponding to the control coefficients optimized from the true shifts, denoted by $A R L_{\tau_{2}}$. Since the CUSUM chart is used to detect the small process shift, we suppose that the value $\tau_{1}=0.95$ is anticipated for the downward chart and the value $\tau_{1}=1.05$ is anticipated for the upward chart while the actual shifts are given in the second column of Table 1 , corresponding to the values of $\tau_{2}$. The large value of $\Delta_{1}$ from this table shows that the performance of the designed charts could be significantly reduced if the anticipated shift is different from the actual shift. For example, if one uses the chart parameters designed for the shift size $\tau_{1}=0.95$ to detect the process shift with the size $\tau_{2}=0.7$, the chart performance will be reduced $\Delta_{1}=93.27 \%$ for the case $\eta=0.2, \theta=0.05, B=1, m=1$ and $n=5$.

In order to overcome this problem, we suggest to evaluate the statistical performance of the CUSUM- $\gamma^{2}$ control chart by using the expected average run length $(E A R L)$ which is defined by

$$
E A R L=\int_{\Omega} A R L \times f_{\tau}(\tau) \mathrm{d} \tau
$$

where $f_{\tau}(\tau)$ is the density function of the shift size $\tau$ over its support $\Omega$.

The design procedure of CUSUM- $\gamma^{2}$ charts is now implemented by finding out the optimial couples $\left(k^{*+}, h^{*+}\right)$ and $\left(k^{*-}, h^{*-}\right)$ satisfying:

- for downward chart,

$$
\left\{\begin{array}{l}
\left(k^{*-}, h^{*-}\right)=\operatorname{argmin}_{\left(k^{-}, h^{-}\right)} \operatorname{EARL}\left(n, m, B, \eta, \gamma_{0}^{*}, \tau, k^{-}, h^{-}\right), \\
A R L\left(n, m, B, \eta, \gamma_{0}^{*}, \tau=1, k^{-}, h^{-}\right)=A R L_{0}
\end{array}\right.
$$


- for upward chart,

$$
\left\{\begin{array}{l}
\left(k^{*+}, h^{*+}\right)=\operatorname{argmin}_{\left(k^{+}, h^{+}\right)} \operatorname{EARL}\left(n, m, B, \eta, \gamma_{0}^{*}, \tau, k^{+}, h^{+}\right), \\
A R L\left(n, m, B, \eta, \gamma_{0}^{*}, \tau=1, k^{+}, h^{+}\right)=A R L_{0} .
\end{array}\right.
$$

By using the measure $E A R L$, the quality practitioners do not need to predetermine a specific value for $\tau$. Instead, they only need to anticipate an interval for $\tau$, which is possible based on their experience in practice. If there is no information about the shape of $f_{\tau}(\tau)$, a uniform distribution, i.e. $f_{\tau}(\tau)=\frac{1}{b-a}$ over the guessed interval $\Omega=[a, b]$, could be applied. In the following, we consider this distribution with two different ranges of the process shift: $\Omega_{D}=[0.5,1)$ and $\Omega_{I}=(1,2]$, corresponding to the decreasing and the increasing case, respectively.

\section{The effect of measurement errors on the CUSUM- $\gamma^{2}$ control charts}

In this section, we show the performance of the proposed CUSUM- $\gamma^{2}$ control chart in the presence of the measurement error. Without loss of generality, the shift in the variance is assumed to be unit, i.e. $b=1$. The in-control value $A R L_{0}$ is set at 370.4. We consider three possible values of $\gamma_{0}$, i.e. $\gamma_{0} \in$ $\{0.05,0.1,0.2\}$ and the sample size $n \in\{5,7,10,15\}$. We also cover in our calculation many scenarios of other parameters, where $\eta \in\{0,0.1,0.2,0.3,0.5,1\}$, $\theta \in\{0.01,0.02,0.03,0.04,0.05\}, B \in\{1,2,3,4,5\}$ and $m \in\{1,3,5,7,10\}$.

Given the values of $m, n, B, \eta, \theta$ and $\gamma_{0}$, the optimal couple $\left(k^{*-}, h^{*-}\right)$ in equation (18) for downward chart and the optimal $\left(k^{*+}, h^{*+}\right)$ in equation (19) for upward chart are solved. These couples define the CUSUM- $\gamma^{2}$ control charts.

Using these optimized coefficients, we evaluate the chart's performance by calculating the out-of-control value $A R L_{1}$. Tables 4-7 present the value of these optimal couples and the corresponding $A R L_{1}$ for some specifice shift sizes. Some conclusions can be drawn from the obtainted results in these tables as follows.

- Given the value of $n, \theta, m, B$ and $\gamma_{0}$ in Table1, the increase of $\eta$ leads to the slight increase of $A R L_{1}$. However, in the case this ratio relatively small, $\eta \leqslant 0.3$, its impact on $A R L_{1}$ are not significant. For example, when $n=7, B=1, \gamma_{0}=0.05, m=1$ and $\tau=1.3$, we obtain $A R L_{1}=12.70$ for $\eta=0$ and $A R L_{1}=12.72$ for $\eta=0.3$.

- Given the value of $n, \eta, m, B$ and $\gamma_{0}$ in Table 2, the lager the value of $\theta$, the larger the value of $A R L_{1}$. That is to say, the the accuracy error has a negative impact on the CUSUM- $\gamma^{2}$ control charts. For example, when $n=5, B=1, \gamma_{0}=0.05, m=1$ and $\tau=0.8$, we have $A R L_{1}=23.52$ for $\theta=0$ and $A R L_{1}=24.54$ for $\theta=0.05$.

- The results in Table 3 say that the rise of $B$ leads to better performance of the CUSUM- $\gamma^{2}$ chart. Take the case $n=7, \eta=0.28, \gamma_{0}=0.2, m=1$ 
and $\tau=0.8$ as an example; for $B=1$ we have $A R L_{1}=18.39$ while $A R L_{1}=17.77$ when $B=5$.

- Table 4 shows the effect of the number of multiple measurements per item on the charts' performance: the larger the value of $m$, the smaller the value of $A R L_{1}$, i.e. the bettter the charts' performance. In general, this result is fit to the finding in Maravelakis ${ }^{12}$ that the multiple measurement is an effective way to compensate for the impact of the measurement error. However, it can be seen from Table 4 that the influence of $m$ on the CUSUM- $\gamma^{2}$ charts' performance is not significant. In most case, the value of $A R L_{1}$ for different values of $m$ changes trivially. For example, with $n=15, \eta=0.28, \gamma_{0}=0.2, B=1$, we have $A R L_{1}=4.09$ for $m=1$ and $A R L_{1}=4.08$ for $m=10$.

- The sample size $n$ has a great impact on the chart performance. When $n$ increases, the $A R L_{1}$ decreases significantly. For example, with $\eta=0.5, \theta=$ $0.05, B=1, m=1, \gamma_{0}=0.1$ and $\tau=0.8$, we have $A R L_{1}=24.59$ when $n=5$ and $A R L_{1}=9.73$ when $n=15$ (Table 4 ).

\section{PLEASE INSERT TABLE 4 HERE \\ PLEASE INSERT TABLE 5 HERE PLEASE INSERT TABLE 6 HERE PLEASE INSERT TABLE 7 HERE}

The effect of the measurement error on the overall performance of the CUSUM- $\gamma^{2}$ control charts measured by $E A R L$ for $n=5$ and $n=15$ is presented in Figures 3-5. In general the obtained results are consistent with those discussed in the previous items. The value of $E A R L$ sharply increases as $\theta$ increases given others parameters. For example, $E A R L=18.4$ for $\theta=0$ while $E A R L=19.3$ for $\theta=0.05\left(n=10, \gamma_{0}=0.2, B=1, m=1\right)$ (Table 6$)$. This tendency is also true for the case of $\eta$, but the effect of $\eta$ on the value of $E A R L$ is weaker than those the effect of $\theta$. In contrast, the increase of $B$ and $m$ leads to a better performance of the proposed charts.

\section{PLEASE INSERT FIGURE 3 HERE \\ PLEASE INSERT FIGURE 4 HERE \\ PLEASE INSERT FIGURE 5 HERE}

In the previous section, we have shown that designing the CUSUM- $\gamma^{2}$ control charts with the optimal parameters corresponding to a specifice shift size may reduce the charts' performance when the acttual shift size is different from the anticipated shift size. This is reflected by the large value of $\Delta_{1}$ in Table 1. In order to evaluate the robustness of the proposed method in which the charts' coefficients are optimized according to the random shift size in an interval, we calculate

$$
\Delta_{2}=\frac{A R L_{\Omega}-A R L_{\tau_{2}}}{A R L_{\tau_{2}}},
$$

where $A R L_{\Omega}$ is the $A R L$ value corresponding to the control coefficients optimized from an interval $\Omega$ of the shift size, and the $A R L_{\tau_{2}}$ is the $A R L$ corresponding to the control coefficients optimized from the true shift $\tau_{2} . \Delta_{2}$ 


\begin{tabular}{c|ccccccc}
\hline Interval & $\tau$ & $\eta=0$ & $\eta=0.1$ & $\eta=0.2$ & $\eta=0.3$ & $\eta=0.5$ & $\eta=1$ \\
\hline \multirow{3}{*}{$\Omega=[0.5,1)$} & 0.5 & 92.07 & 92.01 & 92.08 & 92.07 & 90.79 & 92.43 \\
& 0.7 & 38.47 & 38.45 & 38.47 & 38.48 & 37.64 & 38.53 \\
& 0.8 & 5.35 & 5.35 & 5.35 & 5.35 & 5.04 & 5.35 \\
\hline \multirow{3}{*}{$\Omega=(1,2]$} & 1.3 & 3.17 & 3.16 & 3.12 & 3.12 & 3.17 & 3.16 \\
& 1.5 & 17.75 & 17.74 & 17.65 & 17.65 & 17.74 & 17.65 \\
& 2.0 & 32.37 & 32.36 & 32.24 & 32.24 & 32.32 & 32.11 \\
\hline
\end{tabular}

Table 2: Relative errors $\Delta_{2}$ betwwen the $A R L$ corresponding to the parameters optimized from a predicted interval of the shift size $(\Omega)$ and the actual shift size $\left(\tau_{2}\right)$ under the presence of the measurement error with $B=1, m=1, \theta=0.05$ and $n=5$.

\begin{tabular}{lllllll} 
& & & & & \\
\multirow{2}{*}{0.5} & $(6.4,6.5,7.5)$ & $\theta=0.01$ & $\theta=0.02$ & $\theta=0.03$ & $\theta=0.04$ & $\theta=0.05$ \\
& $(6.8,7.1,8.7)$ & $(6.8,7.1,8.7)$ & $(6.5,6.5,7.5)$ & $(6.6,6.6,7.5)$ & $(6.6,6.6,7.5)$ & $(6.6,6.8,7.6)$ \\
\multirow{2}{*}{0.7} & $(8.8,8.8,10.1)$ & $(8.8,8.9,10.1)$ & $(8.9,7.2,8.7)$ & $(6.9,7.2,8.7)$ & $(6.9,7.27,8.7)$ & $(7.0,7.3,8.7)$ \\
& $(10.0,10.5,13.1)$ & $(10.1,10.5,13.1)$ & $(10.1,10.6,13.1)$ & $(10.0 .9 .0,10.2)$ & $(9.1,9.1,10.2)$ & $(9.1,9.3,10.3)$ \\
\multirow{2}{*}{0.8} & $(16.0,16.1,17.7)$ & $(16.2,16.3,17.8)$ & $(16.4,16.4,18.0)$ & $(16.6,16.6,18.1)$ & $(10.3,10.7,13.2)$ & $(10.4,10.8,13.2)$ \\
& $(20.0,21.0,27.5)$ & $(20.2,21.2,27.6)$ & $(20.4,21.4,27.7)$ & $(20.6,21.6,27.8)$ & $(16.7,16.8,18.2)$ & $(16.9,17.1,18.3)$ \\
\multirow{2}{*}{1.3} & $(11.6,11.7,12.2)$ & $(11.8,11.9,12.4)$ & $(12.0,12.1,12.6)$ & $(12.2,12.3,12.8)$ & $(12.4,12.5,13.8)$ & $(21.0,22.0,27.9)$ \\
\cline { 2 - 7 } & $(10.4,10.6,11.3)$ & $(10.6,10.8,11.5)$ & $(10.8,10.9,11.7)$ & $(11.0,11.1,11.8)$ & $(11.2,11.3,12.0)$ & $(11.4,12.7,13.2)$ \\
\multirow{2}{*}{1.5} & $(5.1,5.1,5.3)$ & $(5.2,5.2,5.4)$ & $(5.2,5.3,5.5)$ & $(5.3,5.4,5.6)$ & $(5.4,5.5,5.7)$ & $(5.5,5.6,5.8)$ \\
& $(4.0,4.1,4.4)$ & $(4.1,4.1,4.4)$ & $(4.2,4.2,4.5)$ & $(4.2,4.3,4.6)$ & $(4.3,4.4,4.7)$ & $(4.4,4.5,4.8)$ \\
\hline \multirow{2}{*}{2.0} & $(2.4,2.4,2.5)$ & $(2.4,2.4,2.6)$ & $(2.5,2.5,2.6)$ & $(2.5,2.5,2.7)$ & $(2.6,2.6,2.7)$ & $(2.6,2.7,2.8)$ \\
& $(1.7,1.8,1.9)$ & $(1.8,1.8,1.9)$ & $(1.8,1.8,2.0)$ & $(1.8,1.9,2.0)$ & $(1.9,1.9,2.0)$ & $(1.9,1.9,2.1)$ \\
\hline
\end{tabular}

Table 3: Compare the performance of CUSUM- $\gamma^{2}$ control charts (first rows) and the EWMA$\gamma^{2}$ control charts (second rows) in the presence of measurement errors for $\eta=0.28, B=1$, $m=1, \gamma_{0}=0.05$ (left side), $\gamma_{0}=0.1$ (middle), $\gamma_{0}=0.2$ (right side) and different values of $\theta$.

represents the relative error between $A R L_{\Omega}$ and $A R L_{\tau_{2}}$. The value of $\Delta_{2}$ for several values of $\tau_{2}$ is presented in Table 2. As can be seen from Table 1 and Table 2 that with the same value of the shift size $\tau_{2}, \Delta_{2}$ is significantly smaller than $\Delta_{1}$. Especially, when the actual shift size is small, say $\tau_{2}=0.8$ or $\tau_{2}=1.3$ in this study, the difference between $A R L_{\Omega}$ and $A R L_{\tau_{2}}$ is quite small. That is to say, the chart parameters optimized from an interval of the shift size can be considered as robust alternatives to the chart parameters optimized from the actual shift size that is not possible to known exactly in practice. This result is similar to the findings in Castagliola et al. ${ }^{1}$.

In addition, it is desirable to make a comparison between the performance of the proposed CUSUM- $\gamma^{2}$ control charts in this study and the EWMA- $\gamma^{2}$ control charts desinged in Tran et al. ${ }^{17}$ under the presence of the measurement error. Table 3 shows the comparison results for the case $m=1, B=1, \eta=$ $0.28, n=5$ and $\theta \in\{0,0.01,0.02,0.03,0.04,0.05\}$.

In fact, this comparison is not really fair since the EWMA- $\gamma^{2}$ control charts in Tran et al. ${ }^{17}$ has been desinged for the specific shift sizes while the CUSUM- $\gamma^{2}$ control charts in our study has been desinged without predetermining a specific shift size. However, the obtained results show that the $A R L_{1}$ value corresponding to the downward CUSUM- $\gamma^{2}$ control chart is smaller than that the $A R L_{1}$ value corresponding to the downward EWMA- $\gamma^{2}$ control chart with the same parameters. That is to say, the downward CUSUM- $\gamma^{2}$ chart outperforms the downward EWMA- $\gamma^{2}$ chart in the presence of the measurement error. Moreover, although the $A R L_{1}$ value corresponding to the upward 
CUSUM- $\gamma^{2}$ control chart is larger than that the $A R L_{1}$ corresponding to the downward EWMA- $\gamma^{2}$ control chart, the difference betwwen them are not significant. Therefore, we can conclude that the proposed method leads to a quite effective performance while its implementation has more practical meaning as a particular shift size is not required.

\section{Application in real industrial scenarios}

Control charts are widely used in a large number of industrial processes where the out-of-control state should be recognized quickly to guarantee the product quality. In order to monitor these processes, the quantity of interest is calcaluted from the collected data, which commonly contain measurement errors. For the processes where the CV squared is considered, that means only $\gamma^{* 2}$ is observable and the true value $\gamma^{2}$ is not obtained. However, by monitoring $\gamma^{* 2}$, one can still detect changes in the actual CV, and then the process variability.

In this section, we present the implementation of the upward CUSUM- $\gamma^{2}$ control chart in the presence of the measurement error. We consider real industrial data from a sintering process in an Italian company that manufactures sintered mechanical parts. These data have been introduced in Castagliola et al. ${ }^{1}$.

According to the description in Castagliola et al. ${ }^{1}$, in order to guarantee a pressure test, the process manufactures parts drop time $T_{p d}$ from 2 bar to 1.5 bar larger than $30 \mathrm{sec}$ as a quality chaxacteristic related to the pore shrinkage. It is stated that the preliminary regression study relating $T_{p d}$ to the quantity $Q_{C}$ of molten copper has demonstrated the presence of a constant proportionality $\sigma_{p d}=\gamma_{p d} \times \mu_{p d}$ between the standard deviation of the pressure drop time and its mean. The quality practitioner then decided to monitor the $\mathrm{CV} \gamma_{p d}=\sigma_{p d} / \mu_{p d}$ to detect changes in the process variability. Based on a root mean square computation, an estimate $\hat{\gamma}_{0}=0.417$ is calculated from a Phase I dataset. The phase II data are reproduced in the first five columns of Table 8.

In Castagliola et al. ${ }^{1}$, the control chart is designed for the specifice shift size $\tau=1.25$ based on the process engineer's experience. However, as analyzed in previous sections, this could seriously affect the statistical run-length properties of the chart. Therefore, in this example, we design the upward CUSUM- $\gamma^{2}$ control chart that optimizes the $E A R L$ over the interval $\Omega=(1,2]$. The control coefficients are found to be $k^{*+}=0.3898930$ and $h^{*+}=12.264137$. Then, we obtain $K^{+}=0.064062$ and $H^{+}=1.910097$. The values of $C_{i}^{*}$ are then presented in the rightmost column in Table 8 and plotted in Figure 2 along with and the control limit $H^{+}$. This figure confirms that from sample \#13 onward, the process is clearly out-of-control. This result is similar to the finding in Castagliola et al. ${ }^{1}$. However, in our implementation, a specifice shift size is not necessary to be predetermined.

PLEASE INSERT TABLE 8 HERE 


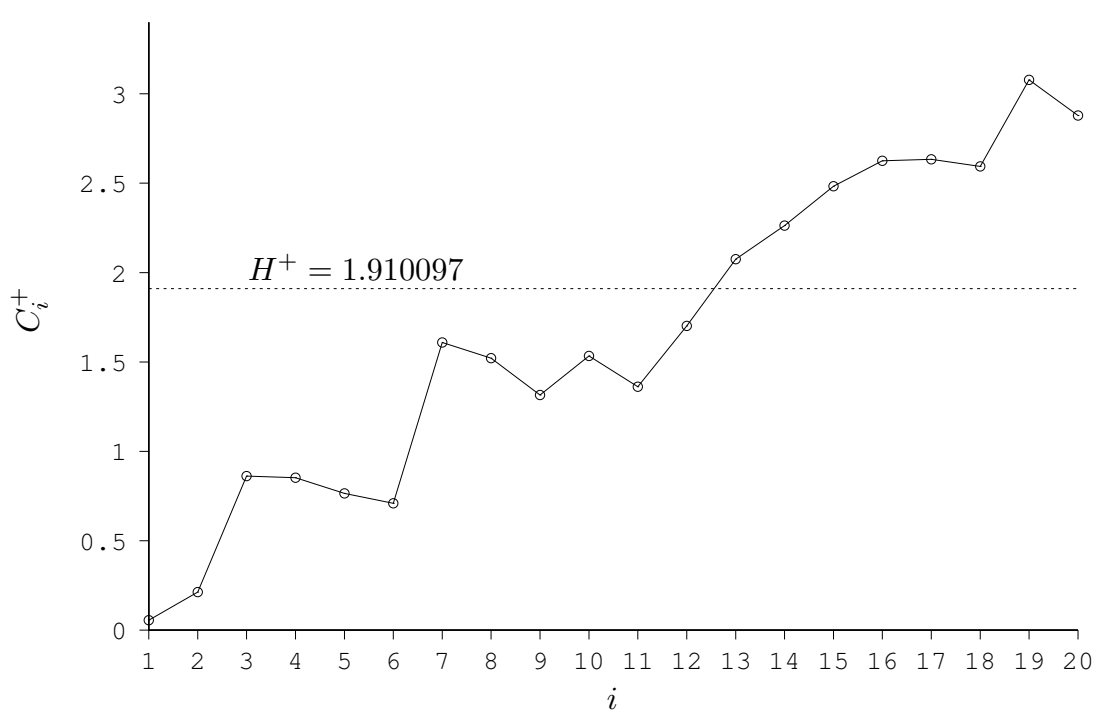

Fig. 2: The upward CUSUM- $\gamma^{2}$ control chart in the presence of the measurement error corresponding to the Phase II data in Table 8.

\section{Concluding remarks}

We have investigated in this paper the effects of the measurement error on the performance of the CUSUM- $\gamma^{2}$ control charts. We have found that the presence of the measurement error have siggnificant effect on the performance of the proposed charts. In particular, both the precision error ratio and the accuracy error have negative impact on the chart performance: the increase of them reduces the power of the CUSUM- $\gamma^{2}$ control charts in detecting changes in the process. Although the increase of the multiple measurement per item leads to the decrease of $A R L_{1}$, its impact is not significant. Therefore, in order to improve the chart performance, one should consider to increase the sample size $n$ or improve the measurement system rather than increasing the number of multiple measurements $m$. Moreover, we have suggested to optimize the chart parameters according to the random shift size in a given interval. An advantage of this method is that it does not require a specifice shift size to be predetermined. The robutness of the proposed method is also pointed out.

\section{Acknowledgments}

The authors thank the anonymous referees for their insightful and valuable suggestions which helped to improve the quality of the final manuscript. 


\section{References}

1. Castagliola, P., Amdouni, A., Taleb, H. and Celano, G. 2011, 'Monitoring the coefficient of variation using ewma charts', Journal of Quality Technology 43(3), 249-265.

2. Kang, C., Lee, M., Seong, Y. and Hawkins, D. 2007, 'A control chart for the coefficient of variation', Journal of Quality Technology 39(2), 151-158.

3. Calzada, M. and Scariano, S. M. 2013, 'A synthetic control chart for the coefficient of variation', Journal of Statistical Computation and Simulation 83(5), 853-867.

4. Castagliola, P., Achoure, A., Taleb, H., Celano, G. and Psarakis, S. 2013, 'Monitoring the coefficient of variation using control charts with run rules', Quality Technology \& Quantitative Management 10, 75-94.

5. Castagliola, P., Achouri, A., Taleb, H., Celano, G. and Psarakis, S. 2013 , 'Monitoring the coefficient of variation using a variable sampling interval control chart', Quality and Reliability Engineering International 29(8), 1135-1149.

6. Amdouni, A., Castagliola, P., Taleb, H. and Celano, G. 2015, 'Monitoring the coefficient of variation using a variable sample size control chart in short production runs', The International Journal of Advanced Manufacturing Technology 81(1-4), 1-14.

7. Castagliola, P., Achouri, A., Taleb, H., Celano, G. and Psarakis, S. 2015, 'Monitoring the coefficient of variation using a variable sample size control chart', The International Journal of Advanced Manufacturing Technology 81(9-12), 1561-1576.

8. Tran, P. H. and Tran, K. P. 2016, 'The efficiency of CUSUM schemes for monitoring the coefficient of variation', Applied Stochastic Models in Business and Industry 32(6), 870-881.

9. Jian, Y., Pingfa, F., Chao, X., Yuan, M. and Shuanggang, H. 2015, 'A novel approach for chatter online monitoring using coefficient of variation in machining process', The International Journal of Advanced Manufacturing Technology 96(1-4), 287-297.

10. Abbasi, S. A. and Adegoke, N. A. 2015, 'Multivariate coefficient of variation control charts in phase I of SPC', The International Journal of Advanced Manufacturing Technology 99(5-8), 1903-1916.

11. Linna, K. and Woodall, W. 20011, 'Effect of measurement error on shewart control chart', Jornal of Quality Technology 33(2), 213-222.

12. Maravelakis, P. 2012, 'Measurement error effect on the CUSUM control chart', Journal of Applied Statistics 39(2), 323-336.

13. Hu, X., Castagliola, P., Sun, J. and Khoo, M. 2015, 'The effect of measurement errors on the synthetic $\bar{X}$ chart', Quality and Reliability Engineering International 31(8), 1769-1778.

14. Noorossana, R. and Zerehsaz, Y. 2015, 'Effect of measurement error on phase II monitoring of simple linear profiles', The International Journal of Advanced Manufacturing Technology 79(9-12), 2031-2040. 
15. Tran, K. P., Castagliola, P. and Celano, G. 2016, 'The performance of the Shewhart-RZ control chart in the presence of measurement error', International Journal of Production Research 54, 7504-7522.

16. Yeong, W., Khoo, M., Lim, S. and Teoh, W. 2017, 'The coefficient of variation chart with measurement error', Quality Technology \& Quantitative Management, 14(4), 353-377.

17. Tran, K. P., Castagliola, P. and Balakrishnan, N. 2018, 'On the performance of coefficient of variation charts in the presence of measurement errors', Quality and Reliability Engineering International 35(1), 329-350.

18. Tran, K. P., Nguyen, H. D., Nguyen, Q. T. and Chattinnawat, W. 2018, One-sided synthetic control charts for monitoring the coefficient of variation with measurement errors, in "2018 IEEE international conference on industrial engineering and engineering management (IEEM)", 1667-1671.

19. Nguyen, H. D., Nguyen, Q. T., Tran, K. P., and Ho, D.P. 2019, 'On the performance of vsi shewhart control chart for monitoring the coefficient of variation in the presence of measurement errors', The International Journal of Advanced Manufacturing Technology, DOI: 10.1007/s00170019-03352-7.

20. Tran, P. H. and Heuchenne, C. 2019, CUSUM control charts for the coefficient of variation with measurement errors, 9th IFAC Conference on Manufacturing Modelling, Management and Control, Berlin, Germany.

21. Breunig, R. 2001, 'An almost unbiased estimator of the coefficient of variation', Economics Letters 70(1), 15-19.

22. Tran, K. P., Castagliola, P., Celano, G., and Khoo, M. B. 2018, 'Monitoring compositional data using multivariate exponentially weighted moving average scheme', Quality and Reliability Engineering International 34(3), 391-402.

23. Brook, D. and Evans, D. 1972, 'An approach to the probability distribution of CUSUM run length', Biometrika 59(3), 539-549.

24. Hawkins, D. and Wu, Q. 2014, 'The CUSUM and the EWMA Head-toHead', Quality Engineering 26(2), 215-222.

25. Iglewicz, B. and Myers, R. 1970, 'Comparisons of approximations to the percentage points of the sample coefficient of variation', Technometrics 12(1), 166-169.

26. Reh, W. and Scheffler, B. 1996, 'Significance tests and confidence intervals for coefficients of variation', Computational Statistics 85 Data Analysis 22(4), 449-452.

27. Vangel, M. 1996, 'Confidence intervals for a normal coefficient of variation', American Statistician 15, 21-26. 


\begin{tabular}{|c|c|c|c|c|c|c|c|}
\hline & $\tau$ & $\eta=0$ & $\eta=0.1$ & $\eta=0.2$ & $\eta=0.3$ & $\eta=0.5$ & $\eta=1$ \\
\hline & \multicolumn{7}{|c|}{$n=5$} \\
\hline$k^{*}$ & & $(0.11,0.10,0.06)$ & $(0.11,0.10,0.05)$ & $(0.11,0.10,0.05)$ & $(0.11,0.10,0.05)$ & $(0.11,0.10,0.05)$ & $(0.11,0.09,0.04)$ \\
\hline \multirow[t]{4}{*}{$h^{*-}$} & & $(6.42,6.54,6.97)$ & $(6.43,6.54,6.97)$ & $(6.42,6.53,6.96)$ & $(6.42,6.53,6.95)$ & $(6.41,6.50,6.90)$ & $(6.38,6.43,6.33)$ \\
\hline & 0.5 & $(10.37,10.45,10.73)$ & $(10.38,10.45,10.73)$ & $(10.37,10.44,10.71)$ & $(10.37,10.43,10.69)$ & $(10.36,10.39,10.58)$ & $(10.30,10.26,9.79)$ \\
\hline & 0.7 & $(14.02,14.12,14.45)$ & $(14.03,14.12,14.45)$ & $(14.02,14.11,14.43)$ & $(14.01,14.09,14.40)$ & $(14.00,14.05,14.28)$ & $(13.94,13.88,13.34)$ \\
\hline & & $(24.54,24.67,25.18)$ & $(24.55,24.67,25.18)$ & $(24.54,24.66,25.16)$ & $(24.53,24.64,25.13)$ & $(24.51,24.59,25.01)$ & $(24.44,24.42,24.07)$ \\
\hline \multirow{5}{*}{$\begin{array}{l}k^{*+} \\
h^{*+}\end{array}$} & & $(0.20,0.21,0.24)$ & $(0.20,0.21,0.25)$ & $(0.20,0.21,0.25)$ & $(0.20,0.21,0.25)$ & $(0.20,0.21,0.26)$ & $(0.20,0.22,0.29)$ \\
\hline & & $(6.08,6.23,6.89)$ & $(6.08,6.24,6.90)$ & $(6.07,6.24,6.93)$ & $(6.08,6.25,6.97)$ & $(6.09,6.28,7.13)$ & $(6.17,6.44,7.92)$ \\
\hline & 1.3 & $(16.46,16.59,17.12)$ & $(16.47,16.59,17.13)$ & $(16.46,16.59,17.15)$ & $(16.46,16.60,17.18)$ & $(16.47,16.63,17.31)$ & $(16.53,16.76,17.91)$ \\
\hline & 1.5 & $(7.22,7.28,7.52)$ & $(7.22,7.28,7.52)$ & $(7.22,7.28,7.53)$ & $(7.22,7.28,7.55)$ & $(7.22,7.29,7.60)$ & $(7.26,7.35,7.87)$ \\
\hline & 2.0 & $(3.40,3.43,3.54)$ & $(3.40,3.43,3.54)$ & $(3.40,3.43,3.54)$ & $(3.40,3.43,3.55)$ & $(3.40,3.44,3.58)$ & $(3.42,3.46,3.70)$ \\
\hline & \multicolumn{7}{|c|}{$n=7$} \\
\hline$k^{*-}$ & & $(0.20,0$ & $0.11)$ & $(0.20,0.19,0.11)$ & $(0$. & $(0$. & $(0.20,0.1$ \\
\hline \multirow[t]{4}{*}{$h^{*-}$} & & $(3.80,3.8$ & $(3.79,3.84,4.63)$ & $(3.80,3.85,4.66)$ & $(3.81,3.93,4.69)$ & $(3.80,3.86,4.80)$ & $(3.82,3.90,4.41)$ \\
\hline & 0.5 & $(6.68,6.71,7.55)$ & $(6.67,6.71,7.56)$ & $(6.68,6.71,7.58)$ & $(6.69,6.81,7.61)$ & $(6.68,6.72,7.72)$ & $(6.69,6.75,7.20)$ \\
\hline & 0.7 & $(9.18,9.22,10.24)$ & $(9.16,9.22,10.24)$ & $(9.18,9.22,10.27)$ & $(9.19,9.33,10.32)$ & $(9.18,9.23,10.45)$ & $(9.19,9.27,9.84)$ \\
\hline & 0.8 & $(16.95,17.01,18.29)$ & $(16.93,17.01,18.30)$ & $(16.95,17.01,18.34)$ & $(16.96,17.14,18.40)$ & $(16.95,17.03,18.58)$ & $(16.96,17.11,18.02)$ \\
\hline \multirow{6}{*}{$\begin{array}{l}k^{*+}+ \\
h^{*+}\end{array}$} & & $(0.22,0.23,0.26)$ & $(0.22,0.23,0.26)$ & $(0.22,0.23,0.26)$ & $(0.22,0.23,0.26)$ & $(0.22,0.23,0.27)$ & $(0.23,0.24,0.30)$ \\
\hline & & $(4.63,4.73,5.15)$ & $(4.63,4.73,5.16)$ & $(4.63,4.74,5.17)$ & $(4.64,4.75,5.20)$ & $(4.64,4.76,5.30)$ & $(4.66,4.87,5.79)$ \\
\hline & 1.3 & $(12.59,12.70,13.16)$ & $(12.59,12.70,13.17)$ & $(12.59,12.70,13.19)$ & $(12.60,12.72,13.22)$ & $(12.60,12.73,13.32)$ & $(12.62,12.85,13.82)$ \\
\hline & 1.5 & $(5.57,5.62,5.83)$ & $(5.57,5.62,5.83)$ & $(5.57,5.62,5.84)$ & $(5.58,5.63,5.86)$ & $(5.57,5.64,5.90)$ & $(5.59,5.69,6.13)$ \\
\hline & 2.0 & $(2.67,2.70,2.80)$ & $(2.67,2.70,2.80)$ & $(2.67,2.70,2.80)$ & $(2.67,2.70,2.81)$ & $(2.67,2.70,2.83)$ & $(2.68,2.73,2.94)$ \\
\hline & \multicolumn{7}{|c|}{$n=10$} \\
\hline$k$ & & $(0.22,0.22,0.18)$ & $(0.2$ & $(0.22,0.22,0.18)$ & $(0$. & 17) & .14) \\
\hline \multirow[t]{4}{*}{$h^{*-}$} & & $(3.00,2$. & $(3.00$ & $(3.00,2.96,3.10)$ & (3. & $(3.0$ & $(3.01,3.03,3.21)$ \\
\hline & 0.5 & $(5.29$ & $(5$. & $(5$. & $(5$ & 35) & $(5.29,5.2$ \\
\hline & 0.7 & $(7.18,7.10,7.24)$ & $(7.18,7.10,7.24)$ & $(7.18,7.10,7.27)$ & $(7.18,7.11,7.24)$ & $(7.18,7.13,7.28)$ & $(7.18,7.19,7.42)$ \\
\hline & 0.8 & $(13.04,12.95,13.21)$ & $(13.04,12.95,13.22)$ & $(13.04,12.95,13.26)$ & $(13.04,12.96,13.23)$ & $(13.04,12.99,13.31)$ & $(13.03,13.08,13.58)$ \\
\hline \multirow{5}{*}{$\begin{array}{l}k^{*+} \\
h^{*+}\end{array}$} & & $(0.25,0.2622,0.28)$ & $(0.25,0.26,0.28)$ & $(0.25,0.26,0.28)$ & $(0.25,0.26,0.28)$ & $(0.25,0.26,0.29)$ & $(0.25,0.27,0.31)$ \\
\hline & & $(3.53,3.57,3.85)$ & $(3.50,3.57,3.85)$ & $(3.53,3.57,3.86)$ & $(3.51,3.58,3.88)$ & $(3.51,3.59,3.94)$ & $(3.54,3.65,4.25)$ \\
\hline & 1.3 & $(9.63,9.68,10.07)$ & $(9.59,9.69,10.07)$ & $(9.63,9.68,10.09)$ & $(9.60,9.70,10.12)$ & $(9.60,9.71,10.19)$ & $(9.64,9.80,10.60)$ \\
\hline & 1.5 & $(4.32,4.34,4.52)$ & $(4.30,4.34,4.52)$ & $(4.32,4.34,4.53)$ & $(4.31,4.35,4.54)$ & $(4.30,4.35,4.57)$ & $(4.32,4.39,4.76)$ \\
\hline & 2.0 & $(2.12,2.13,2.22)$ & $(2.11,2.13,2.22)$ & $(2.12,2.13,2.22)$ & $(2.12,2.14,2.23)$ & $(2.11,2.14,2.25)$ & $(2.12,2.16,2.34)$ \\
\hline & \multicolumn{7}{|c|}{$n=15$} \\
\hline$k^{*-}$ & & $(0.2$ & $(0$. & $5,0.22)$ & & & .19) \\
\hline \multirow[t]{4}{*}{$h^{*-}$} & & $(2.33,2.2)$ & $(2.33,2.2)$ & $(2.33,2.22,2.30)$ & $(2.3$ & $(2.33,2.27,2.32)$ & $(2.20,2.24,2.39)$ \\
\hline & & $(4.12,4.07,4.08)$ & $(4.12,4.07,4$. & $(4.12,4.04,4.08)$ & $(4.12,4.04,4.09)$ & $(4.12,4.07,4.10)$ & $(4.03,4.05,4.14)$ \\
\hline & 0.7 & $(5.55,5.44,5.47)$ & $(5.55,5.44,5.47)$ & $(5.55,5.36,5.47)$ & $(5.55,5.37,5.48)$ & $(5.55,5.44,5.51)$ & $(5.34,5.39,5.61)$ \\
\hline & 0.8 & $(9.88,9.72,9.82)$ & $(9.88,9.72,9.82)$ & $(9.88,9.63,9.84)$ & $(9.87,9.64,9.85)$ & $(9.87,9.73,9.91)$ & $(9.60,9.68,10.11)$ \\
\hline \multirow{5}{*}{$h^{*+}$} & & $(0.29,0.29,0.31)$ & $(0.29,0.29,0.31)$ & $(0.29,0.29,0.31)$ & $(0.29,0.29,0.31)$ & $(0.29,0.29,0.31)$ & $(0.29,0.30,0.33)$ \\
\hline & & $(2.56,2.61,2.79)$ & $(2.56,2.61,2.79)$ & $(2.56,2.60,2.80)$ & $(2.56,2.62,2.81)$ & $(2.57,2.63,2.85)$ & $(2.58,2.67,3.04)$ \\
\hline & 1.3 & $(7.11,7.18,7.49)$ & $(7.11,7.18,7.49)$ & $(7.11,7.18,7.50)$ & $(7.11,7.20,7.52)$ & $(7.12,7.21,7.58)$ & $(7.13,7.28,7.89)$ \\
\hline & 1.5 & $(3.25,3.28,3.42)$ & $(3.25,3.28,3.42)$ & $(3.25,3.28,3.43)$ & $(3.25,3.29,3.44)$ & $(3.25,3.30,3.46)$ & $(3.26,3.33,3.60)$ \\
\hline & 2.0 & $(1.65,1.67,1.74)$ & $(1.65,1.67,1.74)$ & $(1.65,1.67,1.74)$ & $(1.65,1.67,1.75)$ & $(1.65,1.68,1.76)$ & $(1.66,1.69,1.83)$ \\
\hline
\end{tabular}

Table 4: $A R L_{1}$ values of CUSUM- $\gamma^{2}$ control charts in the presence of measurement errors for different values of $\eta, \tau, n$, fixed $\theta=0.05, B=1, m=1, \gamma_{0}=0.05$ (left side), $\gamma_{0}=0.1$ (middle) and $\gamma_{0}=0.2$ (right side). 


\begin{tabular}{|c|c|c|c|c|c|c|c|}
\hline & $\tau$ & $\theta=0$ & $\theta=0.01$ & $\theta=0.02$ & $\theta=0.03$ & $\theta=0.04$ & $\theta=0.05$ \\
\hline$k^{*-}$ & & $(0.11,0.10,0.05)$ & $(0.11,0.10,0.05)$ & $(0.11,0.10,0.05)$ & $(0.110 .10-0.05)$ & $(0.11010-0.05)$ & $(0111010005)$ \\
\hline \multirow[t]{4}{*}{$h^{*-}$} & & $(6.41,6.51,6.92)$ & $(6.42,6.52,6.93)$ & $(6.42,6.52,6.94)$ & $(6.42,6.52,6.94)$ & $(6.42,6.53,6.95)$ & $(6.42,6.53,6.96)$ \\
\hline & 0.5 & $(10.17,10.22,10.44)$ & $(10.21,10.26,10.49)$ & $(10.25,10.31,10.54)$ & $(10.29,10.35,10.60)$ & $(10.33,10.39,10.65)$ & $(10.37,10.44,10.70)$ \\
\hline & 0.7 & $(13.61,13.67,13.93)$ & $(13.69,13.75,14.03)$ & $(13.77,13.84,14.12)$ & $(13.86,13.93,14.22)$ & $(13.94,14.01,14.32)$ & $(14.02,14.10,14.41)$ \\
\hline & 0.8 & $(23.52,23.61,24.04)$ & $(23.72,23.82,24.26)$ & $(23.92,24.02,24.48)$ & $(24.13,24.23,24.70)$ & $(24.33,24.44,24.92)$ & $(24.54,24.65,25.14)$ \\
\hline$k^{*+}$ & & $(0.21,0.22,0.26)$ & $(0.21,0.22,0.25)$ & $(0.20,0.22,0.25)$ & $(0.20,0.21,0.25)$ & $(0.20,0.21,0.25)$ & $(0.20,0.21,0.25)$ \\
\hline \multirow[t]{4}{*}{$h^{*+}$} & & $(6.06,6.241,7.02)$ & $(6.05,6.23,7.00)$ & $(6.06,6.23,7.00)$ & $(6.07,6.24,6.98)$ & $(6.08,6.25,6.97)$ & $(6.08,6.2,6.96)$ \\
\hline & 1.3 & $(15.24,15.38,15.97)$ & $(15.48,15.62,16.21)$ & $(15.72,15.86,16.45)$ & $(15.97,16.10,16.69)$ & $(16.22,16.35,16.93)$ & $(16.47,16.60,17.18)$ \\
\hline & 1.5 & $(6.62,6.68,6.94)$ & $(6.73,6.80,7.06)$ & $(6.85,6.91,7.18)$ & $(6.98,7.04,7.30)$ & $(7.10,7.16,7.42)$ & $(7.22,7.28,7.54)$ \\
\hline & 2.0 & $(3.07,3.09,3.21)$ & $(3.13,3.16,3.28)$ & $(3.20,3.22,3.35)$ & $(3.27,3.29,3.41)$ & $(3.33,3.36,3.48)$ & $(3.40,3.43,3.55)$ \\
\hline & & & & $n=7$ & & & \\
\hline$k^{*-}$ & & $(0.21,0.19,0.10)$ & $(0.21,0.19,0.10)$ & $(0.21,0.19,0.10)$ & $(0.20,0.19,0.10)$ & $(0.20,0.19,0.11)$ & $(0.20,0.18,0.11)$ \\
\hline \multirow[t]{4}{*}{$h^{*-}$} & & $(3.73,3.78,4.76)$ & $(3.74,3.79,4.75)$ & $(3.74,3.80,4.73)$ & $(3.77,3.82,4.71)$ & $(3.79,3.83,4.70)$ & $(3.81,3.93,4.68)$ \\
\hline & 0.5 & $(6.48,6.50,7.55)$ & $(6.51,6.55,7.56)$ & $(6.54,6.59,7.57)$ & $(6.60,6.63,7.58)$ & $(6.64,6.67,7.59)$ & $(6.69,6.81,7.61)$ \\
\hline & 0.7 & $(8.80,8.84,10.11)$ & $(8.88,8.92,10.15)$ & $(8.93,8.99,10.19)$ & $(9.03,9.07,10.23)$ & $(9.11,9.15,10.27)$ & $(9.19,9.33,10.31)$ \\
\hline & 0.8 & $(16.07,16.13,17.74)$ & $(16.24,16.31,17.87)$ & $(16.40,16.48,18.00)$ & $(16.60,16.66,18.13)$ & $(16.77,16.84,18.26)$ & $(16.96,17.14,18.39)$ \\
\hline \multirow{5}{*}{$\begin{array}{l}k^{*+} \\
h^{*+}\end{array}$} & & $(0.23,0.24,0.27)$ & $(0.23,0.24,0.27)$ & $(0.23,0.24,0.27)$ & $(0.23,0.24,0.27)$ & $(0.23,0.23,0.27)$ & $(0.22,0.23,0.26)$ \\
\hline & & $(4.60,4.71,5.22)$ & $(4.60,4.71,5.22)$ & $(4.61,4.72,5.21)$ & $(4.62,4.73,5.21)$ & $(4.62,4.73,5.20)$ & $(4.63,4.74,5.20)$ \\
\hline & 1.3 & $(11.65,11.76,12.29)$ & $(11.83,11.95,12.47)$ & $(12.02,12.14,12.65)$ & $(12.21,12.33,12.84)$ & $(12.40,12.52,13.03)$ & $(12.59,12.71,13.21)$ \\
\hline & 1.5 & $(5.11,5.16,5.39)$ & $(5.20,5.25,5.48)$ & $(5.29,5.34,5.57)$ & $(5.38,5.44,5.67)$ & $(5.48,5.53,5.76)$ & $(5.57,5.63,5.85)$ \\
\hline & 2.0 & $(2.42,2.44,2.55)$ & $(2.46,2.49,2.60)$ & $(2.52,2.54,2.65)$ & $(2.57,2.59,2.70)$ & $(2.62,2.65,2.76)$ & $(2.67,2.70,2.81)$ \\
\hline & & & & $n=10$ & & & \\
\hline$k^{*-}$ & & $(0.22,0.22,0.18)$ & $(0.22,0.22,0.18)$ & $(0.22,0.22,0.18)$ & $(0.22,0.22,0.18)$ & $(0.22,0.22,0.18)$ & $(0.22,0.22,0.18)$ \\
\hline \multirow[t]{4}{*}{$h^{*-}$} & & $(3.01,2.90,3.05)$ & $(3.00,2.92,3.05)$ & $(3.00,2.93,3.06)$ & $(3.00,2.94,3.07)$ & $(3.00,2.96,3.07)$ & $(3.00,2.97,3.08)$ \\
\hline & 0.5 & $(5.20,5.08,5.20)$ & $(5.22,5.11,5.22)$ & $(5.23,5.14,5.24)$ & $(5.25,5.17,5.27)$ & $(5.27,5.21,5.30)$ & $(5.29,5.24,5.33)$ \\
\hline & 0.7 & $(6.97,6.81,6.99)$ & $(7.01,6.87,7.04)$ & $(7.05,6.93,7.08)$ & $(7.10,6.99,7.14)$ & $(7.14,7.05,7.20)$ & $(7.18,7.12,7.25)$ \\
\hline & 0.8 & $(12.46,12.27,12.61)$ & $(12.57,12.41,12.72)$ & $(12.69,12.55,12.85)$ & $(12.81,12.68,12.98)$ & $(12.92,12.83,13.11)$ & $(13.04,12.97,13.24)$ \\
\hline \multirow{5}{*}{$\begin{array}{l}k^{*+} \\
h^{*+}\end{array}$} & & $(0.26,0.26,0.29)$ & $(0.25,0.26,0.29)$ & $(0.25,0.26,0.29)$ & $(0.25,0.26,0.29)$ & $(0.25,0.26,0.28)$ & $(0.25,0.26,0.28)$ \\
\hline & & $(3.47,3.57,3.88)$ & $(3.47,3.56,3.88)$ & $(3.48,3.56,3.88)$ & $(3.49,3.57,3.88)$ & $(3.49,3.57,3.88)$ & $(3.50,3.58,3.88)$ \\
\hline & 1.3 & $(8.87,8.99,9.40)$ & $(9.01,9.12,9.54)$ & $(9.16,9.26,9.68)$ & $(9.30,9.40,9.82)$ & $(9.45,9.55,9.97)$ & $(9.60,9.69,10.11)$ \\
\hline & 1.5 & $(3.94,4.00,4.18)$ & $(4.01,4.06,4.25)$ & $(4.08,4.13,4.32)$ & $(4.16,4.20,4.39)$ & $(4.23,4.28,4.46)$ & $(4.30,4.35,4.53)$ \\
\hline & 2.0 & $(1.92,1.95,2.04)$ & $(1.95,1.98,2.07)$ & $(1.99,2.02,2.11)$ & $(2.03,2.06,2.15)$ & $(2.07,2.10,2.19)$ & $(2.11,2.14,2.23)$ \\
\hline & & & & $n=15$ & & & \\
\hline$k^{*-}$ & & $(0.26,0.25,0.22)$ & $(0.26,0.25,0.22)$ & $(0.26,0.25,0.22)$ & $(0.26,0.25,0.22)$ & $(0.25,0.25,0.22)$ & $(0.25,0.25,0.22)$ \\
\hline \multirow[t]{4}{*}{$h^{*-}$} & & $(2.26,2.21,2.29)$ & $(2.26,2.21,2.29)$ & $(2.26,2.21,2.29)$ & $(2.26,2.21,2.29)$ & $(2.33,2.21,2.29)$ & $(2.33,2.23,2.30)$ \\
\hline & 0.5 & $(4.03,3.99,4.04)$ & $(4.04,3.99,4.04)$ & $(4.05,4.00,4.05)$ & $(4.05,4.01,4.06)$ & $(4.11,4.02,4.07)$ & $(4.12,4.04,4.09)$ \\
\hline & 0.7 & $(5.29,5.19,5.30)$ & $(5.32,5.22,5.33)$ & $(5.35,5.25,5.36)$ & $(5.38,5.28,5.39)$ & $(5.52,5.32,5.43)$ & $(5.55,5.37,5.48)$ \\
\hline & 0.8 & $(9.31,9.18,9.40)$ & $(9.40,9.26,9.48)$ & $(9.48,9.35,9.57)$ & $(9.57,9.43,9.65)$ & $(9.79,9.53,9.74)$ & $(9.88,9.63,9.85)$ \\
\hline$k^{*+}$ & & $(0.29,0.30,0.32)$ & $(0.29,0.30,0.32)$ & $(0.29,0.29,0.32)$ & $(0.29,0.29,0.31)$ & $(0.29,0.29,0.31)$ & $(0.29,0.29,0.31)$ \\
\hline \multirow[t]{4}{*}{$h^{*+}$} & & $(2.54,2.59,2.80)$ & $(2.54,2.59,2.80)$ & $(2.54,2.60,2.79)$ & $(2.54,2.60,2.80)$ & $(2.55,2.61,2.81)$ & $(2.56,2.61,2.81)$ \\
\hline & 1.3 & $(6.58,6.65,6.99)$ & $(6.68,6.76,7.09)$ & $(6.79,6.88,7.19)$ & $(6.89,6.98,7.30)$ & $(7.00,7.08,7.41)$ & $(7.11,7.19,7.51)$ \\
\hline & 1.5 & $(2.99,3.02,3.17)$ & $(3.04,3.07,3.22)$ & $(3.09,3.13,3.27)$ & $(3.14,3.18,3.33)$ & $(3.19,3.23,3.38)$ & $(3.25,3.29,3.43)$ \\
\hline & 2.0 & $(1.51,1.52,1.60)$ & $(1.53,1.55,1.63)$ & $(1.56,1.58,1.66)$ & $(1.59,1.61,1.69)$ & $(1.62,1.64,1.72)$ & $(1.65,1.67,1.75)$ \\
\hline
\end{tabular}

Table 5: $A R L_{1}$ values of CUSUM- $\gamma^{2}$ control charts in the presence of measurement errors for different values of $\theta, \tau, n$, fixed $\eta=0.28, B=1, m=1, \gamma_{0}=0.05$ (left side), $\gamma_{0}=0.1$ (middle) and $\gamma_{0}=0.2$ (right side). 


\begin{tabular}{|c|c|c|c|c|c|c|}
\hline & $\tau$ & $B=1$ & $B=2$ & $B=3$ & $B=4$ & $B=5$ \\
\hline \multicolumn{7}{|c|}{$n=5$} \\
\hline$k^{*-}$ & & $(0.11,0.10,0.05)$ & $(0.11,0.10,0.05)$ & $(0.11,0.10,0.05)$ & $(0.11,0.10,0.05)$ & $(0.11,0.10,0.05)$ \\
\hline \multirow[t]{4}{*}{$h^{*-}$} & & $(6.42,6.53,6.96)$ & $(6.42,6.53,6.96)$ & $(6.42,6.53,6.96)$ & $(6.42,6.53,6.95)$ & $(6.42,6.53,6.95)$ \\
\hline & 0.5 & $(10.37,10.44,10.70)$ & $(10.28,10.34,10.60)$ & $(10.24,10.31,10.57)$ & $(10.23,10.29,10.55)$ & $(10.22,10.28,10.54)$ \\
\hline & 0.7 & $(14.02,14.10,14.41)$ & $(13.82,13.90,14.21)$ & $(13.75,13.83,14.14)$ & $(13.72,13.80,14.11)$ & $(13.70,13.78,14.08)$ \\
\hline & 0.8 & $(24.54,24.65,25.14)$ & $(24.03,24.15,24.63)$ & $(23.86,23.98,24.46)$ & $(23.78,23.90,24.37)$ & $(23.73,23.84,24.32)$ \\
\hline$k^{*+}$ & & $(0.20,0.21,0.25)$ & $(0.20,0.21,0.25)$ & $(0.20,0.21,0.25)$ & $(0.21,0.21,0.25)$ & $(0.20,0.21,0.25)$ \\
\hline \multirow[t]{4}{*}{$h^{*+}$} & & $(6.08,6.25,6.96)$ & $(6.06,6.23,6.93)$ & $(6.06,6.23,6.93)$ & $(6.04,6.23,6.93)$ & $(6.05,6.23,6.93)$ \\
\hline & 1.3 & $(16.47,16.60,17.18)$ & $(15.84,15.97,16.52)$ & $(15.64,15.77,16.32)$ & $(15.53,15.67,16.21)$ & $(15.47,15.61,16.15)$ \\
\hline & 1.5 & $(7.22,7.28,7.54)$ & $(6.91,6.97,7.22)$ & $(6.81,6.87,7.12)$ & $(6.76,6.83,7.06)$ & $(6.73,6.80,7.03)$ \\
\hline & 2.0 & $(3.40,3.43,3.55)$ & $(3.23,3.26,3.37)$ & $(3.18,3.20,3.31)$ & $(3.14,3.18,3.29)$ & $(3.13,3.16,3.27)$ \\
\hline \multicolumn{7}{|c|}{$n=7$} \\
\hline$k^{*-}$ & & $(0.20,0.18,0.11)$ & $(0.20,0.19,0.11)$ & $(0.21,0.19,0.11)$ & $(0.21,0.19,0.11)$ & $(0.21$ \\
\hline \multirow[t]{4}{*}{$h^{*-}$} & & $(3.81,3.93,4.68)$ & $(3.76,3.81,4.68)$ & $(3.75,3.80,4.68)$ & $(3.74,3.79,4.69)$ & $(3.74,3.79,4.69)$ \\
\hline & 0.5 & $(6.69,6.81,7.61)$ & $(6.58,6.60,7.53)$ & $(6.54,6.57,7.51)$ & $(6.53,6.55,7.51)$ & $(6.51,6.54,7.50)$ \\
\hline & 0.7 & $(9.19,9.33,10.31)$ & $(8.99,9.02,10.15)$ & $(8.92,8.96,10.11)$ & $(8.89,8.93,10.09)$ & $(8.87,8.91,10.08)$ \\
\hline & 0.8 & $(16.96,17.14,18.39)$ & $(16.51,16.56,17.99)$ & $(16.36,16.42,17.86)$ & $(16.28,16.35,17.80)$ & $(16.24,16.30,17.77)$ \\
\hline \multirow{5}{*}{$\begin{array}{l}k^{*+} \\
h^{*+}\end{array}$} & & $(0.22,0.23,0.26)$ & $(0.23,0.24,0.27)$ & $(0.23,0.24,0.27)$ & $(0.23,0.24,0.27)$ & $(0.23,0.24,0.27)$ \\
\hline & & $(4.63,4.74,5.20)$ & $(4.61,4.72,5.17)$ & $(4.60,4.71,5.17)$ & $(4.60,4.72,5.17)$ & $(4.60,4.71,5.17)$ \\
\hline & 1.3 & $(12.59,12.71,13.21)$ & $(12.11,12.22,12.70)$ & $(11.95,12.07,12.55)$ & $(11.88,11.99,12.47)$ & $(11.83,11.94,12.42)$ \\
\hline & 1.5 & $(5.57,5.63,5.85)$ & $(5.34,5.39,5.60)$ & $(5.26,5.31,5.53)$ & $(5.22,5.27,5.48)$ & $(5.20,5.25,5.46)$ \\
\hline & 2.0 & $(2.67,2.70,2.81)$ & $(2.54,2.56,2.67)$ & $(2.50,2.52,2.63)$ & $(2.48,2.50,2.60)$ & $(2.46,2.49,2.59)$ \\
\hline \multicolumn{7}{|c|}{$n=10$} \\
\hline$k^{*-}$ & & $(0.22,0.22,0.18)$ & $(0.22,0.22,0.18)$ & $(0.22,0.22,0.18)$ & $(0.22,0.22,0.18)$ & $(0.22,0.22,0.18)$ \\
\hline \multirow[t]{4}{*}{$h^{*-}$} & & $(3.00,2.97,3.08)$ & $(3.00,2.93,3.05)$ & $(3.00,2.92,3.05)$ & $(3.00,2.92,3.05)$ & $(3.00,2.92,3.03)$ \\
\hline & 0.5 & $(5.29,5.24,5.33)$ & $(5.24,5.16,5.25)$ & $(5.23,5.13,5.23)$ & $(5.22,5.12,5.22)$ & $(5.22,5.12,5.21)$ \\
\hline & 0.7 & $(7.18,7.12,7.25)$ & $(7.08,6.95,7.10)$ & $(7.04,6.90,7.06)$ & $(7.02,6.88,7.04)$ & $(7.01,6.87,7.01)$ \\
\hline & 0.8 & $(13.04,12.97,13.24)$ & $(12.75,12.61,12.89)$ & $(12.65,12.50,12.79)$ & $(12.60,12.44,12.74)$ & $(12.58,12.41,12.69)$ \\
\hline \multirow{5}{*}{$\begin{array}{l}k^{* \top} \\
h^{*+}\end{array}$} & & $(0.25,0.26,0.28)$ & $(0.25,0.26,0.28)$ & $(0.25,0.26,0.29)$ & $(0.25,0.26,0.29)$ & $(0.26,0.26,0.29)$ \\
\hline & & $(3.50,3.58,3.88)$ & $(3.48,3.56,3.86)$ & $(3.48,3.56,3.86)$ & $(3.48,3.56,3.86)$ & $(3.47,3.56,3.85)$ \\
\hline & 1.3 & $(9.60,9.69,10.11)$ & $(9.23,9.32,9.73)$ & $(9.12,9.21,9.61)$ & $(9.05,9.15,9.55)$ & $(9.01,9.11,9.50)$ \\
\hline & 1.5 & $(4.30,4.35,4.53)$ & $(4.12,4.16,4.35)$ & $(4.07,4.11,4.29)$ & $(4.03,4.08,4.26)$ & $(4.01,4.06,4.23)$ \\
\hline & 2.0 & $(2.11,2.14,2.23)$ & $(2.01,2.04,2.13)$ & $(1.98,2.00,2.09)$ & $(1.96,1.99,2.08)$ & $(1.95,1.98,2.06)$ \\
\hline \multicolumn{7}{|c|}{$n=15$} \\
\hline$k^{*-}$ & & $(0.25,0.25,0.22)$ & $(0.26,0.25,0.22)$ & $(0.26,0.25,0.22)$ & $(0.26,0.25,0.22)$ & $(0.26,0.25,0.22)$ \\
\hline \multirow[t]{4}{*}{$h^{*-}$} & & $(2.33,2.23,2.30)$ & $(2.26,2.21,2.29)$ & $(2.26,2.21,2.30)$ & $(2.26,2.21,2.26)$ & $(2.26,2.21,2.26)$ \\
\hline & 0.5 & $(4.12,4.04,4.09)$ & $(4.05,4.01,4.05)$ & $(4.04,4.00,4.05)$ & $(4.04,4.00,4.03)$ & $(4.04,3.99,4.02)$ \\
\hline & 0.7 & $(5.55,5.37,5.48)$ & $(5.37,5.26,5.38)$ & $(5.34,5.24,5.37)$ & $(5.33,5.22,5.30)$ & $(5.32,5.22,5.29)$ \\
\hline & 0.8 & $(9.88,9.63,9.85)$ & $(9.53,9.39,9.60)$ & $(9.46,9.32,9.55)$ & $(9.42,9.28,9.45)$ & $(9.40,9.26,9.42)$ \\
\hline \multirow{5}{*}{$\begin{array}{l}k^{*+} \\
h^{*+}\end{array}$} & & $(0.29,0.29,0.31)$ & $(0.29,0.29,0.31)$ & $(0.29,0.29,0.31)$ & $(0.29,0.30,0.32)$ & $(0.29,0.30,0.32)$ \\
\hline & & $(2.56,2.61,2.81)$ & $(2.54,2.60,2.79)$ & $(2.54,2.60,2.78)$ & $(2.54,2.59,2.78)$ & $(2.54,2.59,2.78)$ \\
\hline & 1.3 & $(7.11,7.19,7.51)$ & $(6.84,6.92,7.22)$ & $(6.75,6.84,7.13)$ & $(6.71,6.78,7.08)$ & $(6.68,6.75,7.06)$ \\
\hline & 1.5 & $(3.25,3.29,3.43)$ & $(3.11,3.16,3.29)$ & $(3.07,3.12,3.24)$ & $(3.05,3.08,3.22)$ & $(3.04,3.07,3.21)$ \\
\hline & 2.0 & $(1.65,1.67,1.75)$ & $(1.58,1.60,1.67)$ & $(1.55,1.58,1.64)$ & $(1.54,1.56,1.63)$ & $(1.53,1.55,1.62)$ \\
\hline
\end{tabular}

Table 6: $A R L_{1}$ values of CUSUM- $\gamma^{2}$ control charts in the presence of measurement errors for different values of $B, \tau, n$, fixed $\eta=0.28, \theta=0.01, m=1, \gamma_{0}=0.05$ (left side), $\gamma_{0}=0.1$ (middle) and $\gamma_{0}=0.2$ (right side). 


\begin{tabular}{|c|c|c|c|c|c|c|}
\hline & $\tau$ & $m=1$ & $m=3$ & $m=5$ & $m=7$ & $m=10$ \\
\hline & & & & $n=5$ & & \\
\hline$k^{*-}$ & & $(0.11,0.10,0.05)$ & $(0.11,0.10,0.05)$ & $(0.11,0.10,0.05)$ & $(0.11,0.10,0.05)$ & $(0.11,0.10,0.05)$ \\
\hline \multirow[t]{4}{*}{$h^{*-}$} & & $(6.42,6.53,6.96)$ & $(6.42,6.54,6.97)$ & $(6.42,6.54,6.97)$ & $(6.43,6.54,6.97)$ & $(6.42,6.54,6.97)$ \\
\hline & 0.5 & $(10.37,10.44,10.70)$ & $(10.37,10.45,10.72)$ & $(10.38,10.45,10.72)$ & $(10.38,10.45,10.73)$ & $(10.38,10.45,10.73)$ \\
\hline & 0.7 & $(14.02,14.10,14.41)$ & $(14.02,14.11,14.44)$ & $(14.03,14.11,14.45)$ & $(14.03,14.11,14.45)$ & $(14.03,14.11,14.45)$ \\
\hline & 0.8 & $(24.54,24.65,25.14)$ & $(24.54,24.66,25.17)$ & $(24.54,24.67,25.17)$ & $(24.54,24.67,25.18)$ & $(24.54,24.67,25.18)$ \\
\hline \multirow{5}{*}{$\begin{array}{l}k^{*+} \\
h^{*+}\end{array}$} & & $(0.20,0.21,0.25)$ & $(0.20,0.21,0.25)$ & $(0.20,0.21,0.25)$ & $(0.20,0.21,0.25)$ & $(0.20,0.21,0.25)$ \\
\hline & & $(6.08,6.25,6.96)$ & $(6.08,6.24,6.93)$ & $(6.09,6.23,6.90)$ & $(6.08,6.24,6.90)$ & $(6.08,6.23,6.90)$ \\
\hline & 1.3 & $(16.47,16.60,17.18)$ & $(16.46,16.59,17.14)$ & $(16.47,16.59,17.13)$ & $(16.46,16.59,17.12)$ & $(16.46,16.59,17.12)$ \\
\hline & 1.5 & $(7.22,7.28,7.54)$ & $(7.22,7.28,7.53)$ & $(7.23,7.28,7.52)$ & $(7.22,7.28,7.52)$ & $(7.22,7.28,7.52)$ \\
\hline & 2.0 & $(3.40,3.43,3.55)$ & $(3.40,3.43,3.54)$ & $(3.40,3.43,3.54)$ & $(3.40,3.43,3.54)$ & $(3.40,3.43,3.54)$ \\
\hline & & & & $n=7$ & & \\
\hline \multirow{5}{*}{$\begin{array}{l}k^{*-} \\
h^{*-}\end{array}$} & & $(0.20,0.18,0.11)$ & $(0.20,0.19,0.11)$ & $(0.20,0.19,0$ & $(0.20,0.19,0.11)$ & $(0.20,0.19,0.11)$ \\
\hline & & $(3.81,3.93,4.68)$ & $(3.80,3.86,4.65)$ & $(3.80,3.84,4.63)$ & $(3.80,3.84,4.63)$ & $(3.80,3.84,4.63)$ \\
\hline & 0.5 & $(6.69,6.81,7.61)$ & $(6.68,6.72,7.57)$ & $(6.68,6.71,7.56)$ & $(6.68,6.71,7.56)$ & $(6.68,6.71,7.56)$ \\
\hline & 0.7 & $(9.19,9.33,10.31)$ & $(9.18,9.23,10.26)$ & $(9.18,9.22,10.25)$ & $(9.18,9.22,10.25)$ & $(9.18,9.22,10.24)$ \\
\hline & 0.8 & $(16.96,17.14,18.39)$ & $(16.95,17.03,18.32)$ & $(16.95,17.01,18.31)$ & $(16.95,17.01,18.30)$ & $(16.95,17.01,18.30)$ \\
\hline \multirow{5}{*}{$\begin{array}{l}k^{*+} \\
h^{*+}\end{array}$} & & $(0.22,0.23,0.26)$ & $(0.22,0.23,0.26)$ & $(0.22,0.23,0.26)$ & $(0.22,0.23,0.26)$ & $(0.22,0.23,0.26)$ \\
\hline & & $(4.63,4.74,5.20)$ & $(4.63,4.73,5.17)$ & $(4.63,4.73,5.17)$ & $(4.63,4.73,5.16)$ & $(4.63,4.73,5.15)$ \\
\hline & 1.3 & $(12.59,12.71,13.21)$ & $(12.59,12.70,13.18)$ & $(12.59,12.70,13.18)$ & $(12.59,12.70,13.17)$ & $(12.59,12.70,13.17)$ \\
\hline & 1.5 & $(5.57,5.63,5.85)$ & $(5.57,5.62,5.84)$ & $(5.57,5.62,5.84)$ & $(5.57,5.62,5.83)$ & $(5.57,5.62,5.83)$ \\
\hline & 2.0 & $(2.67,2.70,2.81)$ & $(2.67,2.70,2.80)$ & $(2.67,2.70,2.80)$ & $(2.67,2.70,2.80)$ & $(2.67,2.70,2.80)$ \\
\hline \multirow{6}{*}{$\begin{array}{l}k^{*-} \\
h^{*-}\end{array}$} & & & & $n=10$ & & \\
\hline & & $(0.22,0.22,0.18)$ & $(0.22,0$ & $(0.22,0.22,0.18)$ & $0.18)$ & $(0.22,0.22,0.18)$ \\
\hline & & $(3.00,2.97,3.08)$ & $(3.00,2.95,3.07)$ & $(3.00,2.96,3.06)$ & $(3.00,2.96,3.06)$ & $(3.00,2.96,3.07)$ \\
\hline & 0.5 & $(5.29,5.24,5.33)$ & $(5.29,5.22,5.32)$ & $(5.29,5.23,5.30)$ & $(5.29,5.23,5.31)$ & $(5.29,5.23,5.32)$ \\
\hline & 0.7 & $(7.18,7.12,7.25)$ & $(7.18,7.09,7.24)$ & $(7.18,7.10,7.22)$ & $(7.18,7.10,7.23)$ & $(7.18,7.10,7.24)$ \\
\hline & 0.8 & $(13.04,12.97,13.24)$ & $(13.04,12.93,13.22)$ & $(13.04,12.95,13.20)$ & $(13.04,12.95,13.20)$ & $(13.04,12.95,13.22)$ \\
\hline \multirow{5}{*}{$\begin{array}{l}k^{*+} \\
h^{*+}\end{array}$} & & $(0.25,0.26,0.28)$ & $(0.25,0.26,0.28)$ & $(0.25,0.26,0.28)$ & $(0.25,0.26,0.28)$ & $(0.25,0.26,0.28)$ \\
\hline & & $(3.50,3.58,3.88)$ & $(3.50,3.57,3.86)$ & $(3.53,3.57,3.85)$ & $(3.53,3.57,3.85)$ & $(3.53,3.57,3.85)$ \\
\hline & 1.3 & $(9.60,9.69,10.11)$ & $(9.59,9.69,10.08)$ & $(9.63,9.69,10.08)$ & $(9.63,9.69,10.07)$ & $(9.63,9.69,10.07)$ \\
\hline & 1.5 & $(4.30,4.35,4.53)$ & $(4.30,4.34,4.52)$ & $(4.32,4.35,4.52)$ & $(4.32,4.34,4.52)$ & $(4.32,4.35,4.52)$ \\
\hline & 2.0 & $(2.11,2.14,2.23)$ & $(2.11,2.13,2.22)$ & $(2.12,2.14,2.22)$ & $(2.12,2.13,2.22)$ & $(2.12,2.14,2.22)$ \\
\hline \multirow{6}{*}{$\begin{array}{l}k^{*-} \\
h^{*-}\end{array}$} & & & & $n=15$ & & \\
\hline & & $(0.25,0.25,0.22)$ & $(0.25,0.25,0.22)$ & $(0.25,0.25,0.22)$ & $(0.25,0.25,0.22)$ & $(0.25,0.25,0.22)$ \\
\hline & & $(2.33,2.23,2.30)$ & $(2.33,2.22,2.30)$ & $(2.33,2.27,2.29)$ & $(2.33,2.27,2.29)$ & $(2.33,2.27,2.29)$ \\
\hline & 0.5 & $(4.12,4.04,4.09)$ & $(4.12,4.03,4.08)$ & $(4.12,4.07,4.08)$ & $(4.12,4.07,4.08)$ & $(4.12,4.07,4.08)$ \\
\hline & 0.7 & $(5.55,5.37,5.48)$ & $(5.55,5.35,5.47)$ & $(5.55,5.44,5.47)$ & $(5.55,5.44,5.47)$ & $(5.55,5.44,5.46)$ \\
\hline & 0.8 & $(9.88,9.63,9.85)$ & $(9.88,9.62,9.83)$ & $(9.88,9.73,9.83)$ & $(9.88,9.72,9.83)$ & $(9.88,9.72,9.82)$ \\
\hline \multirow{5}{*}{$\begin{array}{l}k^{*+} \\
h^{*+}\end{array}$} & & $(0.29,0.29,0.31)$ & $(0.29,0.29,0.31)$ & $(0.29,0.29,0.31)$ & $(0.29,0.29,0.31)$ & $(0.29,0.29,0.31)$ \\
\hline & & $(2.56,2.61,2.81)$ & $(2.56,2.60,2.79)$ & $(2.56,2.61,2.79)$ & $(2.56,2.61,2.79)$ & $(2.56,2.61,2.79)$ \\
\hline & 1.3 & $(7.11,7.19,7.51)$ & $(7.11,7.18,7.49)$ & $(7.11,7.18,7.49)$ & $(7.11,7.19,7.49)$ & $(7.11,7.19,7.49)$ \\
\hline & 1.5 & $(3.25,3.29,3.43)$ & $(3.25,3.28,3.42)$ & $(3.25,3.28,3.42)$ & $(3.25,3.29,3.42)$ & $(3.25,3.28,3.42)$ \\
\hline & 2.0 & $(1.65,1.67,1.75)$ & $(1.65,1.67,1.74)$ & $(1.65,1.67,1.74)$ & $(1.65,1.67,1.74)$ & $(1.65,1.67,1.74)$ \\
\hline
\end{tabular}

Table 7: $A R L_{1}$ values of CUSUM- $\gamma^{2}$ control charts in the presence of measurement errors for different values of $m, \tau, n$, fixed $\eta=0.28, \theta=0.05, B=1, \gamma_{0}=0.05$ (left side), $\gamma_{0}=0.1$ (middle) and $\gamma_{0}=0.2$ (right side). 


\begin{tabular}{cccccc}
\hline$i$ & $\bar{X}_{i}^{*}$ & $S_{i}^{*}$ & $\hat{\gamma}$ & $\hat{\gamma}^{* 2}$ & $C_{i}^{*+}$ \\
\hline 1 & 906.4 & 476.0 & 0.525 & 0.27563 & 0.05581 \\
2 & 805.1 & 493.9 & 0.614 & 0.37700 & 0.21300 \\
3 & 1187.2 & 1105.9 & 0.932 & 0.86862 & 0.86181 \\
4 & 663.4 & 304.8 & 0.459 & 0.21068 & 0.85269 \\
5 & 1012.1 & 367.4 & 0.363 & 0.13177 & 0.76465 \\
6 & 863.2 & 350.4 & 0.406 & 0.16484 & 0.70967 \\
7 & 1561.0 & 1562.2 & 1.058 & 1.11936 & 1.60923 \\
8 & 697.1 & 253.2 & 0.363 & 0.13177 & 1.52119 \\
9 & 1024.6 & 120.9 & 0.118 & 0.01392 & 1.31531 \\
10 & 355.3 & 235.2 & 0.662 & 0.43824 & 1.53374 \\
11 & 485.6 & 106.5 & 0.219 & 0.04796 & 1.36189 \\
12 & 1224.3 & 915.4 & 0.748 & 0.55950 & 1.70159 \\
13 & 1365.0 & 1051.6 & 0.770 & 0.59290 & 2.07468 \\
14 & 704.0 & 449.7 & 0.639 & 0.40832 & 2.26319 \\
15 & 1584.7 & 1050.8 & 0.663 & 0.43957 & 2.48295 \\
16 & 1130.0 & 680.6 & 0.602 & 0.36240 & 2.62555 \\
17 & 824.7 & 393.5 & 0.477 & 0.22753 & 2.63327 \\
18 & 921.2 & 391.6 & 0.425 & 0.18062 & 2.59408 \\
19 & 870.3 & 730.0 & 0.839 & 0.70392 & 3.07820 \\
20 & 1068.3 & 150.8 & 0.141 & 0.01988 & 2.87827 \\
\hline
\end{tabular}

Table 8: Illustrative example of Phase II dataset. 


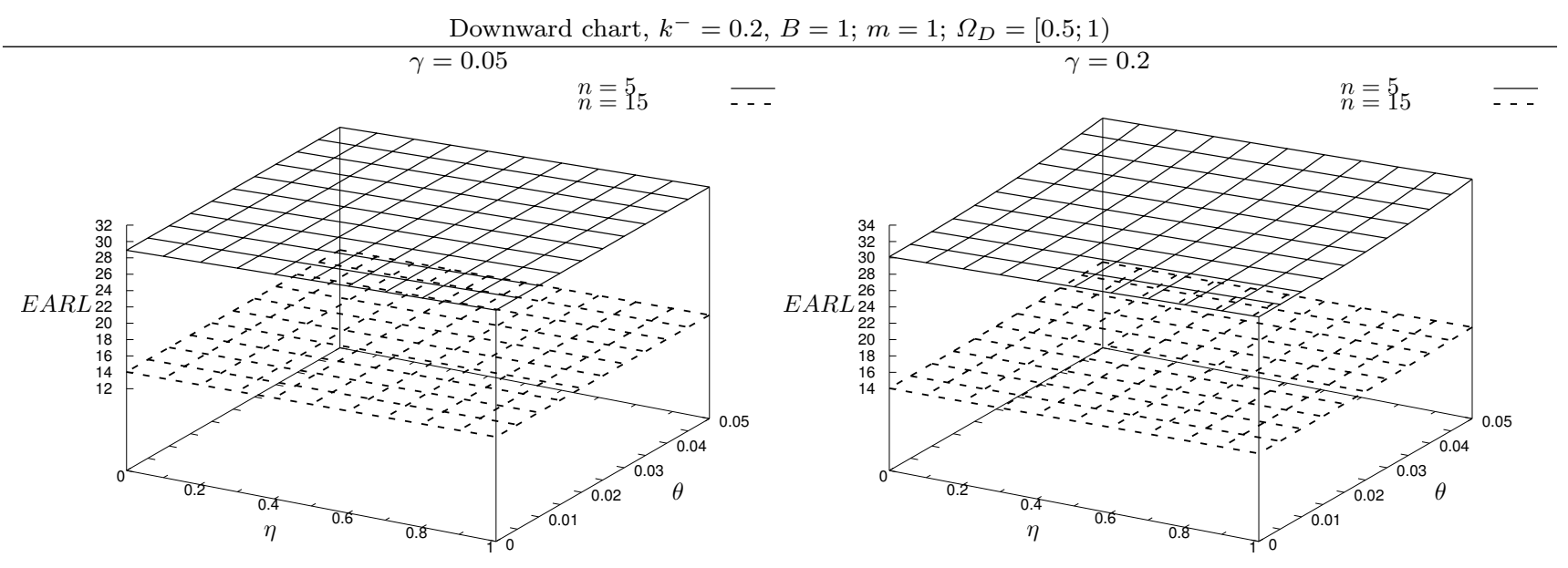

Upward chart, $k^{+}=0.2, B=1 ; m=1 ; \Omega_{I}=(1 ; 2]$

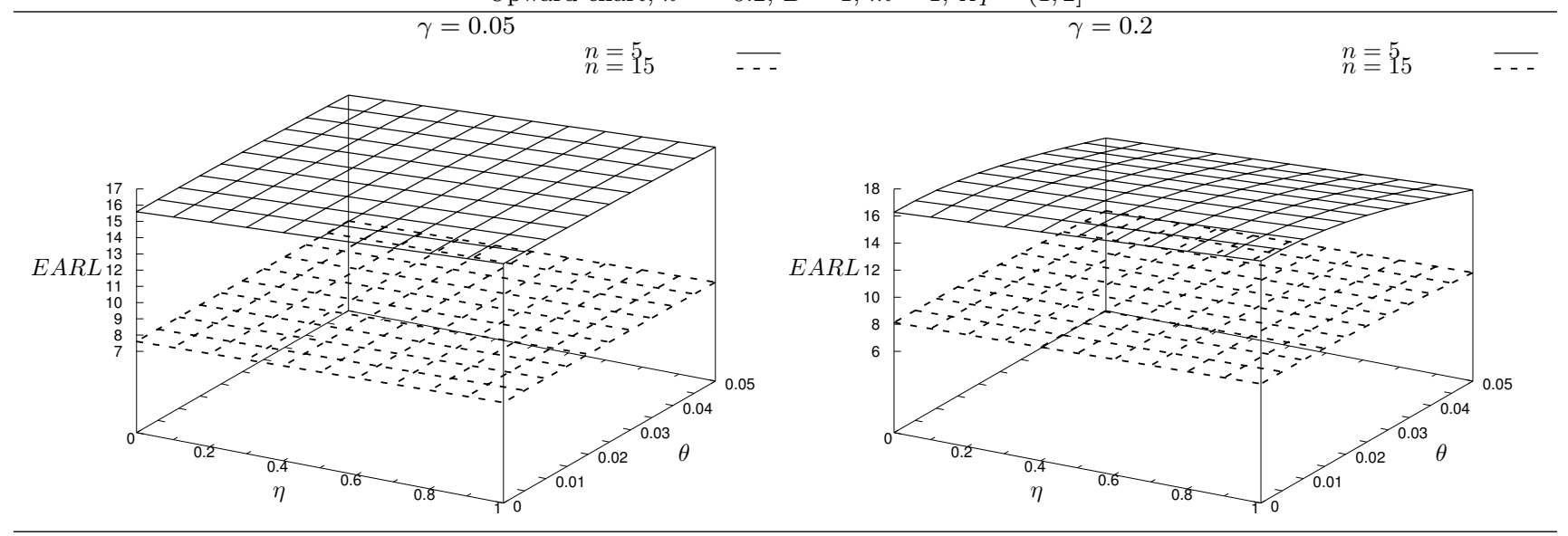

Fig. 3: The effect of $\eta$ and $\theta$ on the overall performance of the CUSUM- $\gamma^{2}$ control charts in the presence of the measurement error. 


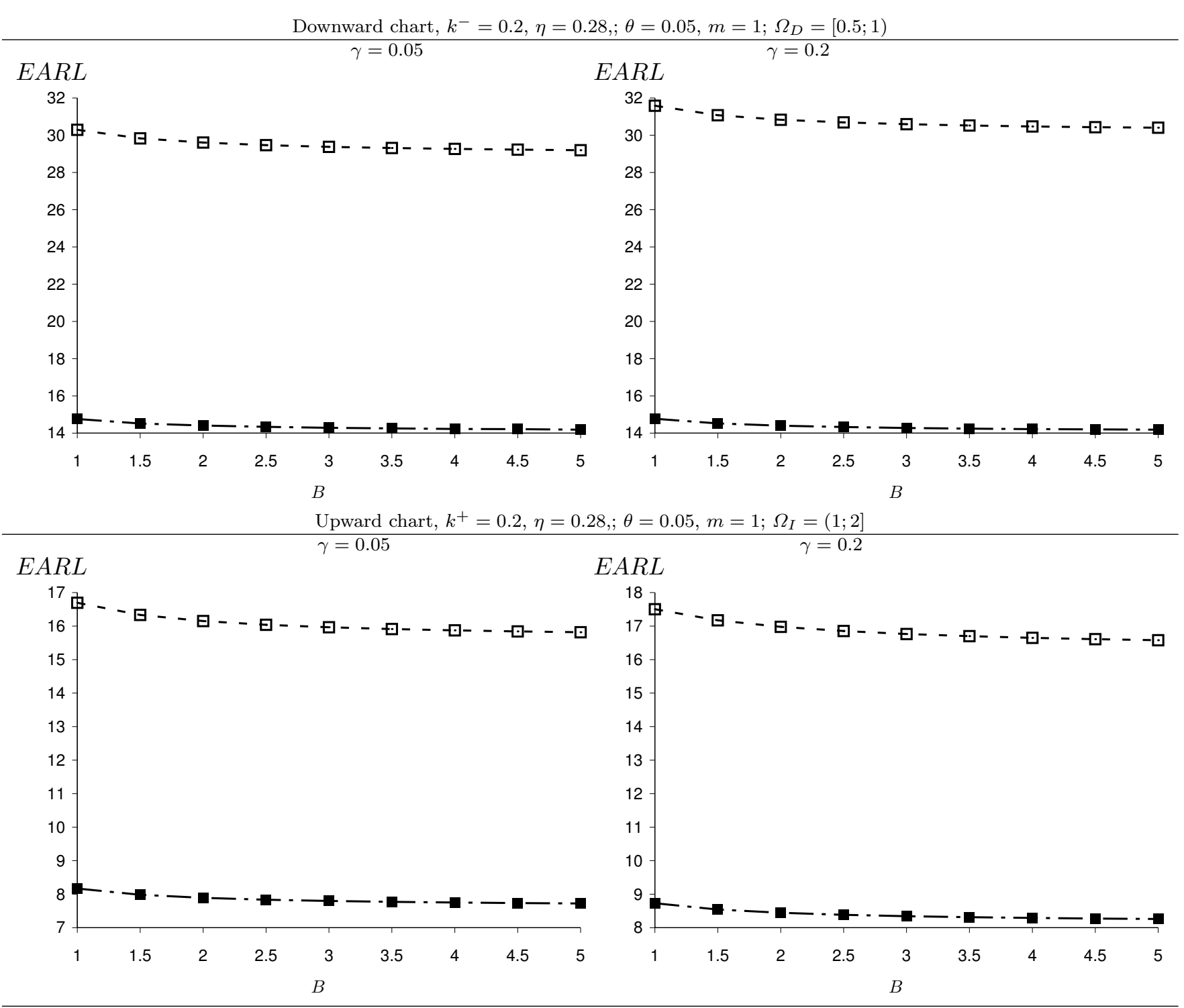

Fig. 4: The effect of $B$ on the overall performance of the CUSUM- $\gamma^{2}$ charts in the presence of the measurement error for $n=5(-\square-)$ and $n=15(-\mathbf{-}-)$ 


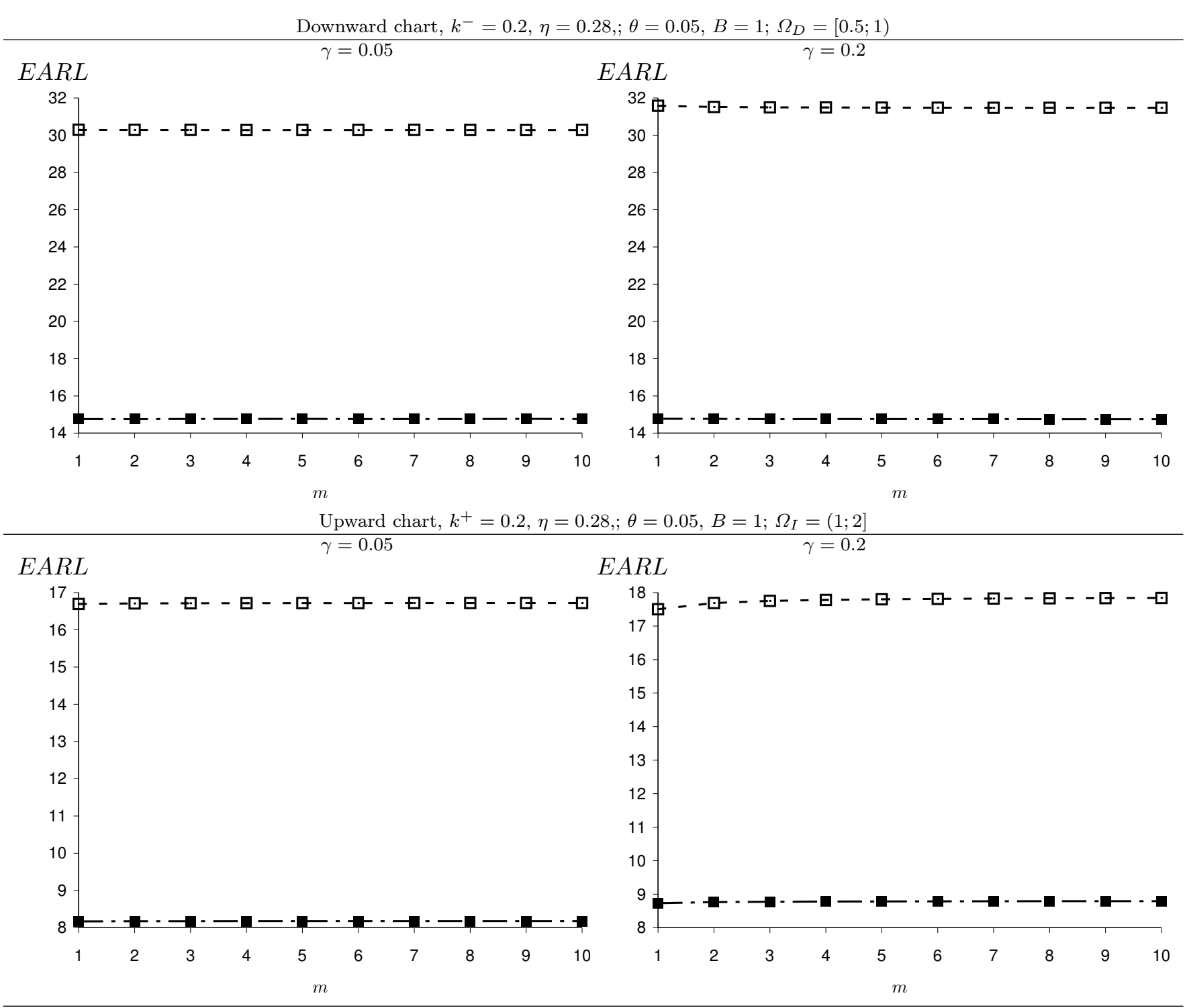

Fig. 5: The effect of $m$ on the overall performance of the CUSUM- $\gamma^{2}$ charts in the presence of the measurement error for $n=5(-\square-)$ and $n=15(-\mathbf{\square}-)$ 